\title{
Innovative Approach Enabled the Retirement of TDRS-1 Compliant with NASA Orbital Debris Requirements
}

Ronald Zaleski, NASA Goddard Space Flight Center, Greenbelt, MD 20771, 301-286-5730, ronald.j.zaleski@ nasa.gov Walter Mirczak, Caelum Research Corp, 419 Via El Chico Redondo Beach, CA 90277, 310-378-8636, Mirczak@ verizon.net Stephen Staich, Legacy Engineering, 411 Susana Avenue Redondo Beach, CA 90277, 310-316-2252, stevestaich@ aol.com Richard Caverly, Orbital Science Corp, NASA GSFC, Greenbelt, MD 20771, 301-286-9606, richard.s.caverly@ nasa.gov Eric Smith, Honeywell Technology Solutions Inc., NASA GSFC, Greenbelt, MD 20769, 301-286-1342, eric.s.smith@ nasa.gov Nicholas Teti, Vertex Aerospace LLC, Queenstown, MD 21658, 301-802-1870, Nicholas.M.Teti@nasa.gov W. Lynn Vaught, General Dynamics C4 Systems, 8201 McDowell Road, Scottsdale AZ, 85257, 575-527-7359, lvaught@ mail.wsc.nasa.gov Dave Olney, NASA Goddard Space Flight Center, Greenbelt, MD 20771, (301) 286-5350, david.j.olney@nasa.gov

Abstract-The first Tracking and Data Relay Satellite (TDRS-1) was deactivated on June $27^{\text {th }} 2010$ following more than 26 years of operation. The end-of-mission (EOM) operations were developed to address the stringent requirements of NPR 8715.6: NASA Procedural Requirements for Limiting Orbital Debris, which consists of three key items: 1) removal from the geosynchronous arc; 2) depletion of the remaining propellant; and 3) passivation of all sources of energy storage or generation [1]. The EOM approach minimized risks while accomplishing these goals. Raising TDRS-1 over $350 \mathrm{~km}$ above geosynchronous was accomplished via proven station change operations. Depleting propellant was the most challenging task, requiring over 20 hours of thruster on-time accumulated within schedule, orbit, and spacecraft subsystem constraints. The attitude configuration and operational procedures, including the unique final passivation method, were thoroughly analyzed and simulated prior to the start of operations. The complete EOM campaign lasted 21 days. The TDRS-1 EOM campaign demonstrated that pre-NPR 8715.6 satellite designs can be made to comply ${ }^{1}$ and that lessons learned could be applied to other satellite designs. The significant TDRS-1 effort demonstrates a commitment by NASA to responsible orbital debris management in compliance with international standards. ${ }^{23}$

\section{TABLE OF CONTENTS}

1. INTRODUCTION. . .1

2. SUMMARY OF CONSTRAINTS AND OPTIONS CONSIDERED. 3

3. PlanNing, ANALYSES, AND SimUlations .......................4 4. ON-ORbit CAMPAign AND PERFormanCE RESUlts ..11 5. CONCLUSIONS . .20

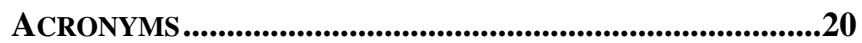
ACKNOWLEDGMENTS ........................................................21

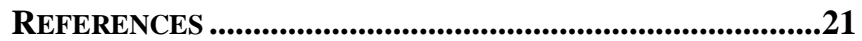
BIOGRAPHY ………........................................................................21

\footnotetext{
${ }^{1}$ A waiver was granted for fuel tank pressurant depletion.

${ }^{2}$ U.S. Government work not protected by U.S. copyright.

${ }^{3}$ IEEEAC paper \#1699, Version 1, Updated October 28, 2010
}

\section{INTRODUCTION}

\section{Spacecraft Description}

The TDRS-1 preliminary design was completed in 1976, and the contract signed for 10 years of fixed-price, leased services from a joint commercial venture originally owned by Western Union. (Subsequently, the contract was fully transferred to NASA.) TDRS-1 development predated any orbital debris considerations, so no EOM passivation capability was provided for the spacecraft. TDRS- 1 is a hexagonal, 3-axis stabilized spacecraft with several protruding booms and antennas (Figure 1).

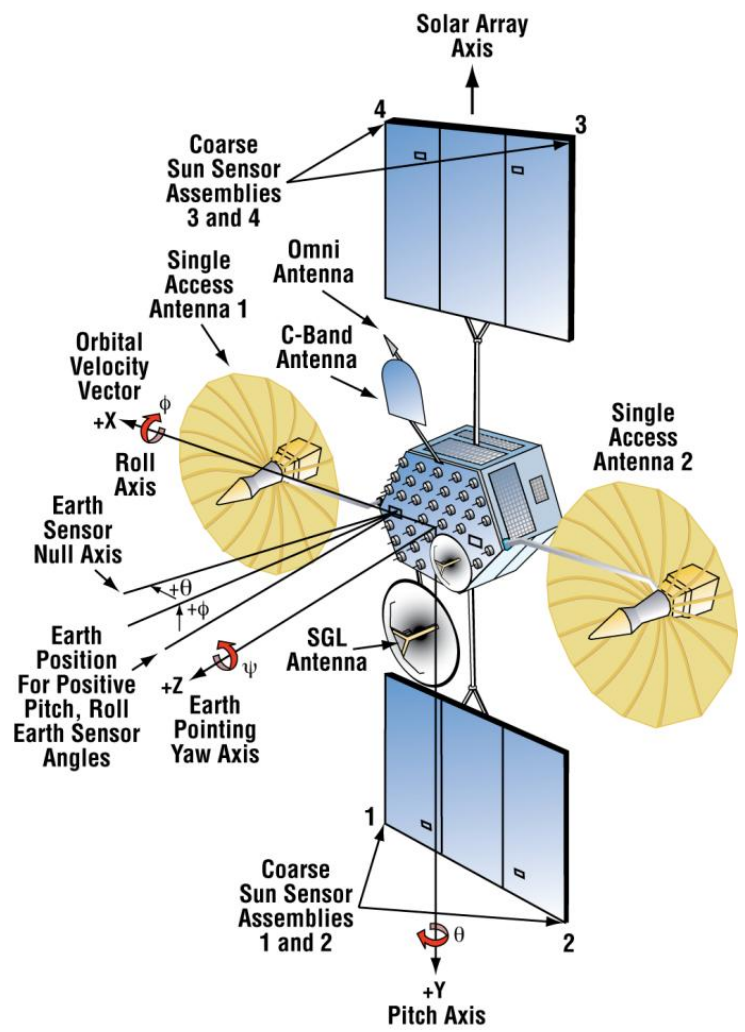

Figure 1 - TDRS-1 On-orbit Configuration

This first generation TDRS spacecraft was built by TRW, Inc. Power was supplied through deployed solar arrays to 
the spacecraft bus; three 24-cell, nickel-cadmium (NiCd) batteries were connected to the bus for eclipse and contingency support. The power bus was unregulated, so when batteries were put online, the bus and payload operated at the unregulated battery voltage.

Although modern for its day, little automation existed onboard TDRS-1. Limited resident modes were available and the capability did not exist to change or upload any new operational modes. Aside from some very limited, hardwired, autonomous power subsystem responses and firmware-sequenced keep-alive Attitude Control Subsystem (ACS) responses, all of the spacecraft functions were commanded from the ground.

Propulsion fuel consisted of hydrazine monopropellant supplied by two tanks within the central hexagonal body. The hydrazine was pressurized with nitrogen using a flexible, diaphragm-type propellant management device. The spacecraft payload consisted of K-band, S-band, and Cband telecommunication services. A space-to-ground link (SGL) K-band dish antenna supported bi-directional user data transmission as well as the telemetry and command (T\&C) necessary for spacecraft operations. A mast-mounted S-band omni antenna supplemented the T\&C functions and was used for loss of attitude, emergency-time-out support, Sun mode, and on-orbit storage.

\section{Brief History}

TDRS-1 was launched on April 4, 1983, from Space Shuttle Challenger (STS-6). During final orbit insertion, the second stage of the Inertial Upper Stage (IUS) experienced a steerable nozzle malfunction resulting in a suspenseful separation from the IUS while tumbling end-over-end at 30 rpm (only minutes of IUS battery life remained at separation), leaving it in a useless transfer orbit [2]. Fortunately, geosynchronous orbit (GEO) was successfully attained using two finger-sized, 4-newton, station-keeping thrusters with fuel originally baselined for 10 years of North/South station-keeping operations.

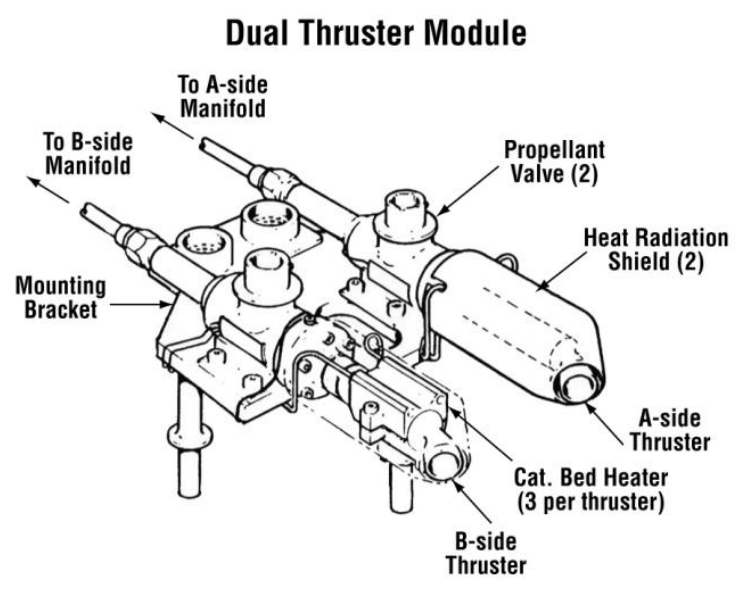

Figure 2-TDRS-1 Dual Thruster Module Assembly
Soon after upper stage separation and attempted Earth acquisition, a thruster-overheating problem specific to TDRS-1 resulted in a suspected hydrazine detonation that ruptured the A-side propulsion line near the negative roll thruster. The spacecraft was designed with a fully redundant propulsion system consisting of 12 A-side and another 12 B-side thrusters, grouped in pairs in dual thruster modules (Figures 2 and 3-North/South thrusters not shown for clarity). Unfortunately, the B-side negative-roll thruster which resides close to the suspected detonation, displayed some performance issues. This event disabled the entire Aside propulsion system and as a precaution the B-side negative roll thruster was also taken out of service. Orbit adjustment maneuvers were developed to compensate for the total loss of negative roll thrusters which enabled a successful TDRS-1 orbit raising to GEO.

ACS thruster overheating remained a problem throughout the life of the spacecraft. Whenever maneuvers required prolonged, low duty-cycle use of the ACS thrusters (i.e., less than $10 \%$ ), elevated thruster temperatures could occur resulting in performance issues and possibly triggering another hydrazine detonation. Thus, thruster thermal issues eliminated the original North-South station-keeping plan, because it required extensive use of ACS thrusters at low duty-cycles. The loss of North-South inclination control at the beginning of life resulted in an abundance of unspent fuel at the end of life, which ultimately had to be disposed of as part of the TDRS-1 EOM activities.

TDRS-1 experienced several anomalies over its service years, but continued to provide communication services in various roles. As inclination increased and additional TDRS were launched, TDRS-1 was assigned to the National Science Foundation (NSF) in 1989, primarily supporting South Pole stations. (Its high inclination provided good polar visibility.) NSF support lasted until 21 October 2009 when the last of six traveling wave tube (TWT) assemblies wore out, precluding the satellite's ability to transfer user data. All T\&C was then supported via the low-bandwidth, S-band, omni antenna. Because of this inability to support users, TDRS-1 was decommissioned on 28 October 2009 and subsequently moved to a temporary orbital slot of $56.5^{\circ} \mathrm{W}$ longitude to await disposal.

\section{Challenges to Fulfilling the Orbital Debris Requirements}

TDRS-1 was not designed to meet current orbital debris requirements, but the EOM effort did satisfy all but one of the debris requirements defined by NASA-STD-8719.14 Handbook for Limiting Orbital Debris [3]. The handbook defined two broad criteria: raising the orbit such that it would remain at or above GEO $+200 \mathrm{~km}(35,986 \mathrm{~km})$ altitude for a period of at least 100 years, and passivation of all stored energy sources. Using spacecraft system design manuals and schematics, a designated TDRS-1 EOM team implemented capabilities that were never envisioned in the original spacecraft design. Taking advantage of the abundance of fuel along with the multitudes of 
configurations and ground-commanded cross-strap connections, procedures were developed to:

(1) Raise altitude to a targeted $350 \mathrm{~km}$ above GEO.

(2) Deplete fuel while maintaining the orbit approximately $350 \mathrm{~km}$ above GEO (minimizing the impact of fuel depletion to the $350 \mathrm{~km}$ above GEO orbit proved very challenging).

(3) Spin down the momentum wheel while maintaining attitude.

(4) Permanently discharge the battery.

(5) Permanently remove the battery from the bus.

(6) Permanently turn off any RF radiation sources.

(7) Permanently disable all active RF receivers.

Pressurant passivation was the only orbital debris requirement that was not met because the propellant tank was designed with a diaphragm which prevented the nitrogen pressurant from being vented at EOM. A waiver was granted for this requirement.

This paper describes the analyses, approaches, and on-orbit execution of these EOM activities.

\section{SUMMARY OF CONSTRAINTS AND OPTIONS CONSIDERED}

\section{Key Constraints}

Delta Velocity Maneuver Burn Time-The TDRS-1 thrusters were subject to a unique phenomenon (corrected on subsequent spacecraft) referred to as "thruster choking." Thruster choking reduces the flow of propellant through a thruster, both diminishing its control authority and also causing a rise in temperature because less heat is transferred away by the propellant flow. The condition limited TDRS-1 thruster operations to $100 \mathrm{~s}$ or less for most of its operational life. A re-evaluation [4] determined that $600 \mathrm{~s}$ was a safe limit for TDRS-1 propulsion system conditions in delta- $\mathrm{V}$ mode. With an estimated $22 \mathrm{~h}$ of thruster burn time required to deplete the remaining propellant, the 10min burn constraint would dictate that 132 such burns be performed. A combination of thruster thermal diurnal variation and the optimal orbital position for burn execution (i.e., apogee or perigee) would further dictate that the burns be separated by 12 or $24 \mathrm{~h}$.

Qualification Limit of Thruster Burn Duration-The TDRS-1 thrusters had undergone ground qualification testing for a maximum duration of $3 \mathrm{~h}$. While this was far in excess of the 600-s on-orbit burn limit, the qualification limit would become a factor as other fuel depletion approaches were considered.

Eclipse Season-All TDRS-1 EOM activities were required to be completed before the start of the fall eclipse season, which began on 11 July 2010. Any TDRS-1 attitude recovery procedure would have involved use of a Sunpointing mode, which could have been impacted by an eclipse. In addition, as various fuel depletion modes were considered, positive electrical power balance also became a concern.

Over-the-horizon (OTH) Operations-Ground sites other than the White Sands Complex (WSC) usually experienced significant command loss and telemetry dropouts when communicating with TDRS-1, because of lower uplink signal strength and/or less optimal ground site location. EOM activities were constrained to a direct link with WSC which effectively imposed a deadline on completion, because a westward longitudinal drift of TDRS-1 would begin as soon as its semi-major axis was increased above the geosynchronous value. Based on the nominal orbitraising plan, it was estimated that TDRS-1 would drift out of view to $\mathrm{WSC}\left(165^{\circ} \mathrm{W}\right.$ sub-satellite longitude point) on 4 July 2010. EOM had to be completed before this time. It was too high of a risk to allow the single event upset (SEU) susceptible TDRS-1 to drift completely around the Earth with spotty communications coverage and resume EOM operations as it drifted back into view from the East.

\section{Fuel Depletion Options Considered}

It was recognized early on that the first priority was raising the TDRS-1 orbit to a debris-compliant super-synchronous altitude. To minimize the risks during this phase of the EOM, the 600-s burn duration limit was adopted. Additionally, maneuvers began after the spring eclipse season ended and when thruster thermal conditions were more favorable. As a result of these constraints, orbit raising could not start until 5 June 2010 and would not complete until 13 June 2010. The remaining EOM activities, fuel depletion, and passivation were constrained to start after June $13^{\text {th }}$ and complete before TDRS-1 loss of view from WSC. This constraint heavily impacted the viability of the various fuel depletion options considered. Prior to orbit raising, TDRS-1 was estimated to have $139.47 \mathrm{~kg}$ of propellant. The orbit raising used $13.06 \mathrm{~kg}$ of propellant leaving an estimated $126.41 \mathrm{~kg}$ that needed to be depleted by fuel depletion activities.

Delta Velocity Maneuvers-The most straightforward approach to fuel depletion was to consider maneuvers already used on TDRS-1 (East/West delta-velocity maneuvers) or used on other TDRS (North/South deltavelocity maneuvers). East/West maneuvers, in particular, were a routine operation during the TDRS-1 mission. The time window available, due to the eclipse and OTH constraints combined with the 600-s burn duration constraint, eliminated East/West or North/South maneuvers as a viable option to achieve complete fuel depletion. Allowing for two 600-s burns per day would require over 60 days to realize fuel depletion. This clearly exceeded the time window available. 
Ground-commanded Thruster Firings - To better utilize the time window available, some thought was given to openloop thruster pulsing - an operation that would be similar to the routinely performed momentum unloads [5]. Given the depletion time window, this method would have required 1098 command sequence transmissions per day, or 46/h. This approach was deemed operationally impractical, as it required near-continuous command activity for approximately 17 days in order to expend the remaining propellant.

Propellant Venting from A-side Leak-Consideration was given to utilizing the ruptured A-side propulsion system [6] to deplete the fuel. This approach sought to vent hydrazine from each propellant tank into space by opening the isolation valve connecting that tank with the A-bank of thrusters. The approach was attractive because it depleted fuel with little or no impact to the orbit (due to the low exit velocity of the propellant through the leak), and it was an efficient method of removing propellant from the spacecraft ( $0.54 \mathrm{~kg}$ could be expended per minute). Given this rate, the total residual propellant mass could be removed with approximately $233 \mathrm{~min}$ of isolation valve open time. However, several unknowns about the leak and the valves were considered serious risks [5]. These unknowns included: the ability to control spacecraft attitude while hydrazine vented out the ruptured propellant line; the potential for hydrazine to freeze in the propellant line(s) and at the thruster valve; the ability of the isolation valves to support the required cycling; and the potential for fuel detonation in the propellant line due to the "water hammer" effect. Ultimately, the risk was considered too great given the unknowns; any on-orbit testing could only be safely performed after reaching super-synchronous altitude thus posing a risk to the schedule.

Z-spin-Another approach to deplete fuel was to fire the station-keeping thrusters (e.g., Z1 and Z2) while spinning the spacecraft about its Z-axis (see Figure 3). The thruster pair torques would approximately cancel each other while the spin would serve to stabilize attitude and average out most of the delta-velocity imparted to the orbit. The depletion burns could be performed without any active thruster attitude control thus eliminating the thruster choking issue (i.e., no thrusters operate in problematic duty cycle regions) that limited normal $\Delta v$ maneuver durations. Two orientations of the Z-axis were considered.

(1) Z-spin, Sun-pointed-From an electrical power perspective, having the Z-axis Sun-pointed is an attractive orientation. The solar arrays can be positioned to receive full Sun continuously, similar to normal operations. The communications geometry would not always be favorable since the hemispherical omni antenna would spend half the orbit pointing away from Earth. Rather than lose communications for half an orbit, an attitude maneuver would need to be performed twice per orbit around spacecraft 6 a.m. and 6 p.m. to keep the omni in view of WSC. The maneuver would serve to pitch the spacecraft $180^{\circ}$, maintaining good communication for the next halforbit and also slewing the solar arrays to maintain Sun pointing. These half-orbit maneuvers added operational complexity and also posed some risk of thruster choking while being executed.

(2) Z-spin Orbit Normal-This Z-axis spin approach would orient the spacecraft $+Z$-axis in the direction of the orbit normal before introducing a negative spin rate. While solar array power input would be reduced and the communications link would operate near the edges of the omni antenna pattern, this approach was selected as the best compromise. A complete description is given in a following section.

\section{Planning, Analyses, and Simulations}

\section{Orbit Raising}

The highest priority was to remove the spacecraft from GEO. Successfully boosting the spacecraft to a sufficiently high altitude offered the potential of isolating the spacecraft such that TDRS-1 would not pose a significant risk to other spacecraft in the geosynchronous arc [7].

Based upon guidance presented in NASA-STD-8719.14 [3], the minimum safe perigee for a spacecraft with TDRS-1's physical properties (i.e., spacecraft area, mass, and reflectivity) was calculated to be $290 \mathrm{~km}$ above GEO (for the 100-year requirement). Given that the intent was to dispose of approximately $126 \mathrm{~kg}$ of hydrazine via thruster firings, once the disposal orbit altitude had been attained, the decision was made to target a perigee altitude of GEO plus $350 \mathrm{~km}$. Although the methodology developed to execute the propellant disposal was intended to limit the effect upon the orbit, it was still anticipated that significant orbit lowering could occur.

The increase in orbital altitude would require a change in velocity of $12.6 \mathrm{~m} / \mathrm{s}$, or a total burn duration of $7239 \mathrm{~s}$. Given the constraints of the TDRS-1 thrusters, such a change in orbit was not a trivial undertaking. Prior to Fall 2009, station-keeping maneuvers had been specifically limited to $100 \mathrm{~s}$ due to concerns about thruster performance during longer duration burns. ${ }^{4}$ Repositioning maneuvers of 900, 480, and $320 \mathrm{~s}$ were performed in 1990, 1994, and 1996 respectively. The requirement to de-orbit TDRS-1 provided an impetus to re-examine this conservative limitation.

The limitation of burn duration was based upon the assumption, and confirmed with empirical experience, that following burn termination a significant amount of thruster

\footnotetext{
${ }^{4}$ These concerns centered primarily around two TDRS-1 RCS limitations: 1) the loss of the primary system and the negative roll thruster on the redundant system, due to thruster failures during the rescue mission (1983), and 2) the potential for the occurrence of thruster "choking" during extended thruster operation. This phenomenon of choking had the effect of reducing thruster impulse by as much as $90 \%$, thus seriously compromising attitude control during critical operations.
} 
firing using hot thrusters was often required to dampen attitude errors and rates prior to transition to ACS Normal Mode (i.e., reaction wheel control) operation. An analysis of the $320 \mathrm{~s}$ duration burns executed in 1996 indicated, however, that given a sufficient amount of time in the burn (at least 6-7 min), the ACS control law would effectively dampen all attitude errors and rates. The attitude rates at burn termination would thus be effectively nulled, allowing for an immediate transition to Normal Mode operation with little or no thruster firings. The burn duration length was still limited by other spacecraft operational risks ${ }^{5}$, which dictated a need to allow for some thruster use capability at burn termination. The burns had to be of sufficient duration to allow for operation of the ACS control law to dampen attitude rates, but not long enough for thruster temperatures to become problematical for use following burn termination. Analysis of the 1996 relocation burns and subsequent simulation suggested that a burn duration of $10 \mathrm{~min}$ would be sufficient to achieve attitude error and rate damping while posing an acceptable level of operational risk.

When TDRS-1 was decommissioned, the temporary move to the non-operational location $\left(56.5^{\circ} \mathrm{W}\right)$ provided an opportunity to validate the 600 -s burn duration analysis.

Accordingly, the two start drift/relocation burns were each $10 \mathrm{~min}$ in duration and used the same thrusters planned for the final orbit raising. Attitude and thruster thermal performance during these burns validated the analysis. Based upon this performance, planning for the final orbit raising during TDRS-1 EOM projected the use of twelve 10 -min burns to achieve the necessary orbital velocity change.

\section{Selected Fuel Depletion Approach - Z-spin Orbit Normal}

Overview and Concerns-The Z-spin orbit normal configuration has the principal axis (i.e., maximum moment of inertia) spinning normal to the orbit plane. This option had the best combination of low risk, time to complete, minimal procedural complexity, and spacecraft stability. This approach did pose its own risks and concerns. However, sufficient planning time was available to adequately determine the risks and develop contingency approaches should those risks materialize. None of the possible risks were found to be insurmountable. The spacecraft systems concerns associated with the selected approach were:

1) Would there be enough electrical power to operate the necessary spacecraft equipment in this configuration? The solar arrays would be fixed, relative to the spacecraft body,

\footnotetext{
5 The TDRS-1 onboard processor memory was not hardened. As a consequence, it experienced periodic Single Event Upsets in RAM. An upset to a critical ACS control law parameter could be problematical during thruster control operations. There was also the potential for erratic gyro operation. Such anomalous operation occurred infrequently (three times during previous maneuver operations), but necessarily involved a requirement for manual correction of the situation (i.e., in this case, cycling gyro power)
}

hence the power generating solar cells would be cycling in and out of sunlight. The arrays would be in effect generating approximately a third of their normal solar power.

2) Would there be reliable, continuous space-to-ground communications through the omni antenna at a marginal orientation with respect to the ground station? Would there be combinations of body spin angle and orbital position (latitude) that could block the command and telemetry path? This was particularly worrisome because the spacecraft would need to receive commands every $51 \mathrm{~s}$ during the burns to continue firing the thrusters. Any burn interruption would result in an undesired attitude disturbance.

3) Would the spacecraft be in an acceptable state of thermal balance? The unusual orientation relative to the Sun, the spinning motion, and the non-nominal suite of powered equipment required careful analysis. This issue was coupled with power concerns and an uncertainty in heater power consumption.

4) Would Z-spin be dynamically stable while thrusting, and would operators have adequate knowledge of the inertial orientation of the angular momentum vector and be able to control it if required? The orientation of the angular momentum vector would not be directly measurable. The concern arose out of the fact that the Sun sensors on the solar arrays can measure the inertial orientation of the spin axis in only one direction (relative to the Sun). However, the clock-angle of the spin vector about the Sun line would not be directly measurable - thereby introducing an uncertainty in the knowledge of the tilt.

This paper addresses all of these concerns, first from the perspective of planning and preparing for the EOM campaign followed by a report on the actual on-orbit performance results.

\section{Rotational Dynamics of Z-spin Orbit Normal Approach}

Analytical Discussion - The principal axes of a body are determined by the mass properties; the orientation is such that two of the axes are in direction of maximum and minimum moments of inertia. TDRS- 1 is designed so that the principal axes are nearly aligned with the body axes with the largest moment of inertia very nearly aligned with the spacecraft Z-axis. A well-known property of spinning bodies is that rotation about the axis with the maximum moment of inertia is stable and will continue without change in the absence of external torques (i.e., between fuel depletion burns). Ideally then, performing a fuel depletion burn while spinning about the Z-axis will not cause the spin axis to change orientation in space.

TDRS-1's four Z-axis thrusters are shown in Figure 3. The Z-axis thrusters are used either in coupled pairs or individually for yaw attitude control, or in thrust pairs for $\Delta \mathrm{v}$. Used in thrust pairs, they ideally would balance the torques generated about the spacecraft pitch, yaw and roll control axes resulting in a pure force through the spacecraft center of gravity. Actually, the torque cancellation is not 
complete, leaving residual torques about two of the axes which will result in perturbations to the spin during the fuel depletion burns.

A residual torque about the $\mathrm{Z}$-axis will cause the spin speed to either decrease or increase. Therefore, adjustments to the spin speed would need to be made during the fuel depletion burn in order to keep the spin speed close to the desired rate.

Spin speed adjustments could be made by off-modulating one of the two $\mathrm{Z}$-axis thrusters resulting in a temporary imbalance of the thruster torques to correct the spin speed. Although the residual torque about roll is relatively small, the residual torque about the pitch is significant and will perturb the orientation of the spin vector. There are two effects of this external torque: nutation and precession.

Nutation is the motion of a spinning body's principal axis about the angular momentum vector in inertial space. The nutation angle is the instantaneous angle between the principal axis and the angular momentum vector. Precession is the change in the direction of the angular momentum vector in inertial space and is always due to an external torque.

In nutation, the body will spin/rotate faster than the spin axis nutates — the exact ratio determined by the body mass properties. After a complete nutation period the spin axis retraces the motion in space, however, the body axes will differ from the previous nutation motion due to the difference between the spin rate and the nutation rate. For TDRS-1's mass properties, this ratio is approximately 4:3after four spin periods and three nutation periods, the spacecraft body will return to almost the same position it was in at the beginning of the periods.

If an applied external disturbance torque is constant throughout the many rotations, then the spin vector would experience a periodic combination of nutation and precession (the bounds determined by the size of the disturbance torque, the spin rate, and the ratio of the transverse products of inertia with the maximum inertia). Termination of the disturbance torque at the end of the burn will cease the periodic precession motion, but will cause the spin vector to start a different (i.e., torque-free) nutation path about the precessed mean orientation.

Since the amount of nutation and precession resulting from a disturbance torque is inversely proportional to the spin rate, stability is better at a high rotation rate. However, if the spacecraft $\mathrm{Z}$-axis tilt away from orbit normal is large, then the omni antenna view of the ground station will begin to be blocked by spacecraft structure at certain times of the day. In these situations, reacquiring a command link is more difficult at a higher spin rate. Simulations indicated that a spin rate of $1.0 \%$ was an acceptable compromise between spin stiffness and communications integrity.
The final precession and residual nutation can be controlled/minimized by carefully timing the fuel depletion burns to end when nutation and spin have brought the Z-axis nearly back to where it was before the burn started. Thus, all fuel depletion burns were planned in increments of 4 spin periods (approximately 3 nutation periods). Because the selected spin rate was $1.0 \%$, the burn durations were planned for multiples of approximately $24 \mathrm{~min}$. The spacecraft body axes will not return exactly to the original position at the start of the burn. This offset will increase with each 24-min cycle, and will have a noticeable effect on residual nutation and precession with the long burns $(>1$ hour) planned for the fuel depletion activity.
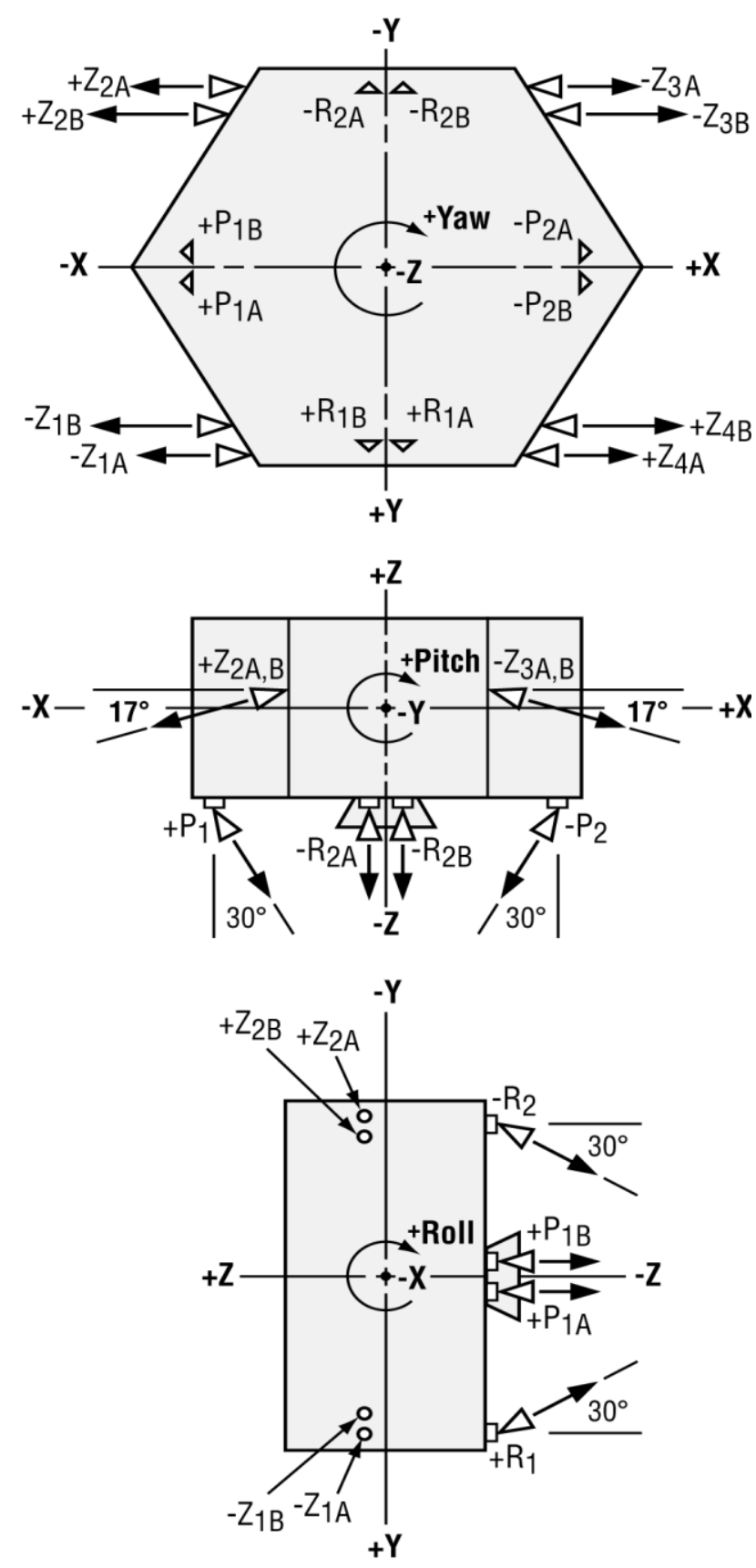

Figure 3 - Orientation of Thrusters Relative to TDRS Reference Axes 
Simulation of Dynamics - To predict attitude dynamic behavior, a computer model was developed that could simulate and display the motion of a rotating rigid body subject to thruster torques. Assuming that appendage flexibility and fuel slosh are negligible, the dynamics are governed by Euler's equations for rigid body motionconsisting of three non-linear scalar differential equations, one for each of the principal axes. The non-linearity is a consequence of the non-inertial body frame. In the simulation the equations are integrated to give angular rates as a function of time, based on inputs specified for each principal axis. The inputs included the moments of inertia, initial angular rate, and torque as a function of time. Multiplying the output for each axis by the corresponding principal moment of inertia gave the corresponding angular momentum component as a function of time in the body frame.

It was also useful to transform body components of these and other vector quantities into inertial coordinates and vice versa. This required an additional set of differential equations that operated on the angular velocity time functions to produce coordinate transformations (a function of time) in the form of Euler parameters (quaternions). Their initial values, in effect, selects the inertial coordinate system; but for the most part, the two systems were made coincident at $\mathrm{t}=0$. The results were then converted to directional cosine matrices.

Because the solar torque, the dominant external disturbance at GEO, was significantly smaller than the torques from the thrusters, only torques from the two thrusters used, Z1 and $\mathrm{Z} 2$, were included. All the equations were programmed into MATLAB ${ }^{\circledR}$ as a Simulink model. ${ }^{6}$

A globe of unit radius displayed on a MATLAB graphics window was generated to represent either a body or inertial frame of reference. Using spherical coordinates, time functions of vectors were normalized to unit magnitude and plotted on the globe's surface. This provided insight when examining simulation results.

Figure 4a shows the typical trajectory of the angular velocity and angular momentum in the body frame over a 600 -s thruster firing. The center of the coordinate system corresponds to the $-\mathrm{Z}$ principal axis. Markers indicate 1-min time intervals starting from the origin at $\mathrm{t}=0$. At this time, a constant transverse torque along $+\mathrm{Y}$ was applied causing both the angular momentum and angular velocity to leave the origin and orbit counterclockwise from the center of the figure along offset elliptical trajectories that cycle in 8-min intervals (the nutation period). When the torque was removed at $600 \mathrm{~s}$, both quantities switch to more

\footnotetext{
${ }^{6}$ Trade names and trademarks are used in this report for identification only. Their usage does not constitute an official endorsement, either expressed or implied by the National Aeronautics and Space Administration.
}

pronounced elliptical trajectories centered about the origin; this is torque-free body nutation. (Had the thrust continued six more minutes in this simulation case, the trajectory would have returned to the origin and the final nutation would have been near zero.)

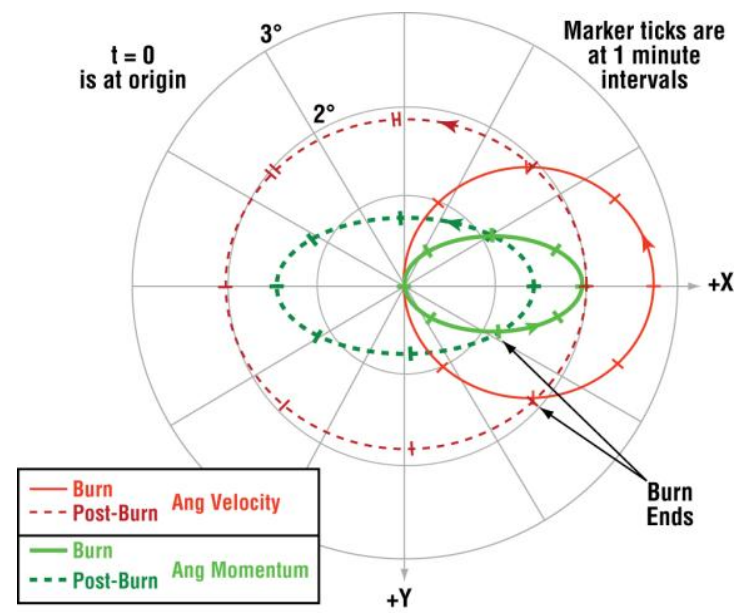

Figure 4a-Typical Trajectories of the Angular Velocity and Angular Momentum in the Body Frame.

Figure $4 \mathrm{~b}$ shows the same case as Figure $4 \mathrm{a}$, with angular momentum and the body $-\mathrm{Z}$ axis plotted in the inertial coordinate system coinciding with the body coordinates at $\mathrm{t}=0$. Both quantities start at the origin. Note that the circular motion of the angular momentum orbits over a 6-min interval (the spin period). The $-\mathrm{Z}$-axis direction coincides with the angular momentum direction every 8 -min (nutation cycle). This is indicated at the points where the cusps of the body Z-axis curve (Burn portion) touch the angular momentum path in Figure 4b. Also note that the post-burn angular momentum ceases to precess as shown by the marker on the angular momentum path at burn end in the same figure.

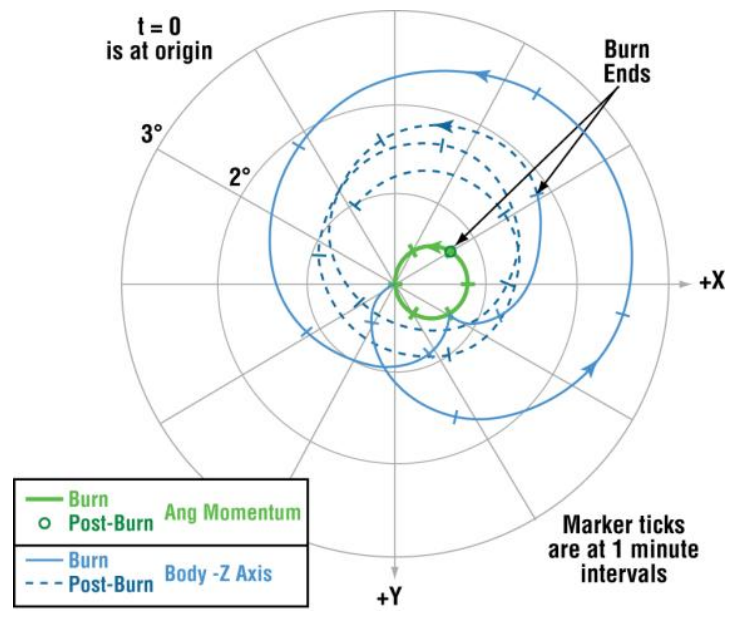
Figure 4b-Typical Trajectories of the Angular Momentum and Body $-Z$ (+ Spin) Axis in Inertial Coordinates




\section{Impact on Super-synchronous Orbit}

NASA's Goddard Space Flight Center (GSFC), Flight Dynamics Facility (FDF) evaluated the effect of fuel depletion burns in the Z-spin orbit normal orientation. The evaluation was divided into the effects of thrust components along the orbit normal and those in the orbit plane.

Thrust in Orbit Plane-FDF evaluated the effect on apogee/perigee altitude of thrust deviation from orbit normal at two burn positions in the orbit, the ascending and descending orbit nodes. The thrust deviations from orbit normal were applied in each of four principle in-plane directions (separated by $90^{\circ}$ ). Only the in-plane thrust component was modeled, with its size defined by a thrust magnitude and the direction of the deviation from orbit normal. Thrust in the orbit normal direction was neglected. Off-pointing angles of $5^{\circ}$ and $10^{\circ}$ and burn durations of 1 and $8 \mathrm{~h}$ were evaluated. Each of the tested directions produced a change in apogee/perigee heights. Figure 5 shows the results for a 1 -h burn with $10^{\circ}$ of off-pointing. The worst-case off-pointing direction lowered perigee by almost $20 \mathrm{~km}$. The maximum change to either apogee or perigee was $2 \mathrm{~km} / \mathrm{h}$ of thrusting per degree off orbit normal. The actual in-plane thrust was not sufficient to separate these effects from other assumption-related perturbations.

Outcome-Due to the initial uncertainty of TDRS-1's spin axis orientation it was decided to make the first burn at perigee. This minimized the chances of lowering the orbit before the spacecraft orientation could be determined. The apsis sensitivities discussed in the preceding paragraph coupled with the low predicted offset of the Z-axis from orbit normal before the first burn lent confidence to the approach of commencing the burns with one of 72 minutes duration.

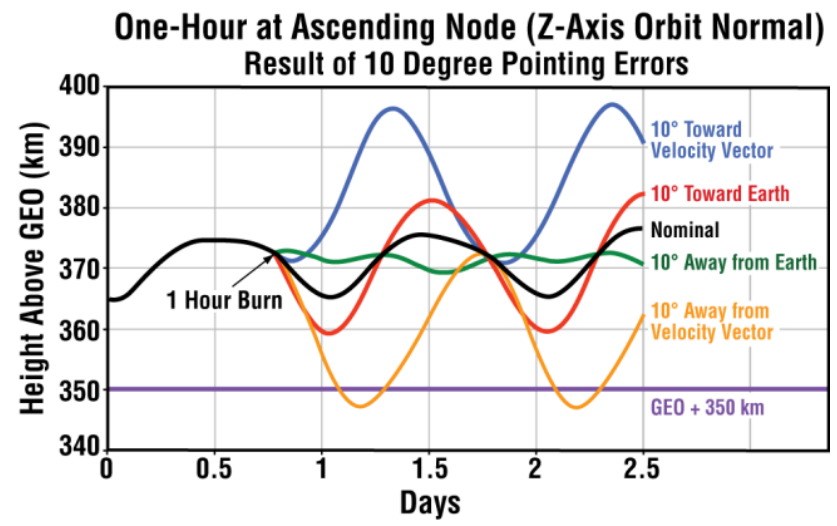

Figure 5 - One-hour Burn with $10^{\circ}$ Off-pointing

\section{Propellant Estimate}

At the start of the orbit-raising operation, the estimated remaining hydrazine propellant was $139.47 \mathrm{~kg}$. Of that total, $66.60 \mathrm{~kg}$ was estimated to remain in Tank A and $72.87 \mathrm{~kg}$ in Tank B. Since there was no direct readout of remaining propellant mass on the spacecraft, these estimates were based upon approximately 27 years of methodical bookkeeping. Given that there were numerous sources of error for this method, not least of which was the fact that the on-board processor truncated the telemetered thruster ontime, the expectation was that the estimates would only be accurate to within $10-20 \mathrm{~kg}$.

\section{Tank Management}

Because of certain concerns relating to possible thruster performance during the final stage of fuel depletion ${ }^{7}$, the decision was made to manage tank usage carefully such that final depletion would occur on both tanks simultaneously. Thus, Tank A (the lower pressure tank) was selected as the initial tank on which fuel depletion operations would begin. Once the estimated mass in Tank A reached approximately $20 \mathrm{~kg}$, a switch would be made to Tank B, followed by a resumption of fuel depletion operations. At the point at which the estimated Tank B mass approximately equaled that in Tank A, both tanks would be configured to the thruster bank (i.e., both tank isolation valves opened), the tank pressures equalized, and fuel depletion operations resumed to completion.

\section{Tank Pressure Characteristics Near Depletion}

Introduction-The Z-spin approach required the careful orchestration of events while the tanks expended their last few kilograms of propellant. The Z-spin orientation needed to be maintained after "depletion" to support the final passivation activities. This required that the final burn be terminated before complete depletion (since erratic thruster performance could cause an unrecoverable attitude disturbance), and also that sufficient propellant remained to perform nutation control as well as to de-spin the spacecraft to $0.5 \%$. The EOM team needed a predictive characterization of tank pressure behavior as the final kilograms of propellant are forced out of the tanks in order to establish on-orbit burn stop criteria. To characterize the in-flight pressure changes that may occur when the two spacecraft tanks begin to empty of hydrazine, a test was performed on a spare TDRS tank at GSFC.

Discussion-Test data showed that if the amount of gas trapped under the diaphragm due to hydrazine decomposition is insignificant, then there could be three or more minutes of available fuel flow from the start of rapid pressure decline until the flight tanks are depleted. This would provide sufficient response time to terminate the final depletion burn and avoid an attitude disturbance. Armed with this knowledge and assuming the gas trapped under the diaphragm was small but not zero, the White Sands EOM team planned the final depletion burn to terminate when the pressure decreased to 100 psi.

\footnotetext{
7 The concern specifically related to the expectation that thruster performance could become erratic during this final stage of fuel depletion. That is, it was speculated that dissolved nitrogen gas, coming out of solution and into bubbles as tank pressure decreased, would begin to displace liquid hydrazine at the thruster injector valve. In a worst case scenario, such an effect could also damage the thrusters. In such an event, fuel depletion operations might have to be terminated.
} 


\section{Power Management}

The Z-spin orientation with fixed solar array positioning provided intermittent solar array power only. The focus of the electrical power planning was to anticipate an expected marginal power situation, given the reduced power and the mandatory bus loads required to operate the spacecraft. Onorbit tests at GEO were conceived, e.g., rotating the arrays $360^{\circ}$, which would have allowed characterization of the power subsystem in conditions similar to a Z-spin. The concern of stranding the spacecraft at GEO because of some anomaly during testing precluded their execution. Analysis was the only means available to help anticipate power management issues and plan work-around procedures. Of particular concern was charging batteries with the solar arrays paddle-wheeling in and out of the Sun, producing power similar to a half-wave rectified sine-wave. With a 6min spin period, a little less than 3 min was available for the solar arrays to recharge the batteries while supporting all critical bus loads.

The spacecraft's Electrical Power Subsystem (EPS) was designed with a 37-volt (at 73 watts) high-rate battery charge solar array section, a 37-volt (at 16 watts) battery trickle-charge section, and a 25-volt (at 1700 watts) array section for bus and payload operation. With only $3 \mathrm{~min}$ available for battery recharge, it was clear that neither the trickle-charge nor the high-rate battery charge solar array sections would be sufficient to return the power removed during the 3-min out-of-Sun period. Routinely, the batteries are disconnected from the bus when Sun is on the array to allow the batteries to charge and float at a voltage higher than the bus (via the 37-volt battery charge sections). Since solar array voltage increases as the load current decreases and solar array voltage also increases with reduced solar array temperatures, it was hypothesized that the higher voltage and charge current necessary to charge the batteries could be attained from the 25-volt bus operations section of the array. With "cool" solar arrays and sufficient load reduction, under this hypothesis, the spacecraft power operating point would move to a higher voltage operating point on the solar array IV (current-voltage) curve resulting in the 25- volt solar array section providing sufficiently high voltage to support battery charging.

One difficulty in power planning resulted from the large uncertainty in end-of-life solar array degradation; no good assessment was possible to determine if the voltage necessary to recharge batteries could be provided from the 25-volt solar array section. Using the limited on-orbit data, solar array IV curve analysis indicated that the 1700-watt array section operating at normal temperatures would not produce the voltage necessary for battery charging; but this analysis did not take into account the uncertainties of the expected solar array aging or lower solar array temperatures (from the paddle-wheeling of the arrays in the Sun).

Another challenge in planning resulted from the equipment never having been shut off for the life of the spacecraft resulting in uncertainties in determining true bus unit electrical loads. The spacecraft power subsystem manual typically listed unit load demands on the high end. The lack of data, in combination with the problem in determining changes in unit/panel heater demands while in the flat spin, made it difficult to accurately characterize load savings.

Uncertainty in the load assessment dictated the development of a flexible load shedding and modulation plan. Using a listing of non-critical loads sorted by estimated wattage, loads could be progressively shed or added, as needed. It was thought that loads would have to be shed down to the essential units from the likely limited array voltage available for battery charge. To track battery state-of-health during Zspin operations, a process was developed to integrate the battery charge/discharge currents and monitor individual cell voltages. In the unlikely event of higher than expected voltage from the solar array, a load modulation plan was also available in order to maintain the bus voltage within acceptable limits and avoid battery overcharge or undercharge.

Despite the unknowns, the consensus was that the electrical power subsystem could be reasonably expected to support the Z-spin approach. A contingency procedure was available to command TDRS-1 to Sun mode and recharge the batteries in the event of abnormally low battery state-ofcharge, which would increase the operational complexity of the approach but was only needed as a contingency.

A 20-hour period was allocated following the initiation of the Z-spin to achieve thermal equilibrium, evaluate subsystem performance, and allow an assessment of all the unknowns.

\section{Thermal Control}

The TDRS-1 thermal control system (TCS) was designed to maintain all spacecraft equipment within acceptable temperature limits throughout all mission phases. This was accomplished primarily by passive means utilizing combinations of thermal hardware, which included multilayer insulation, second surface mirror radiators, and thermal control coatings. These elements are supplemented by thermostatically-controlled heater circuits.

The reaction control system (RCS) also utilized thermostatically controlled heaters located on the propellant feed lines, propellant tanks, dual thruster module (DTM) valves, fill and drain module (FDM), and the propellant distribution module (PDM) in order to maintain propellant temperatures above freezing $\left(4 \cdot 4^{\circ} \mathrm{C}\right)$.

In support of the TDRS-1 EOM, the TDRS-3 Thermal Math Model (TMM) was modified to represent the TDRS-1 configuration. The TDRS-1 thermal model was then correlated to TDRS-1 October 2009 and March 2010 flight data. To account for differences between the TDRS-1 and TDRS-3 thermal model configurations, several 
modifications were made to properly represent the propellant thrusters, thermal coating properties, multi-layer insulation (MLI) degradation, and the $\mathrm{NiCd}$ battery radiators.

Several iterations were made using the TDRS-1 Thermal Desktop $^{\text {TM } 8}$ (Earth-pointing) thermal model to obtain good correlation between the TDRS-1 thermal model and flight data. The temperature comparison data for the positive pitch thrusters shown in Figure 6 is typical of how well the thermal model predications correlate to the flight temperatures. This comparison was done for the thrusters, batteries, propulsion tanks, solar arrays, and other temperature critical payload and spacecraft components needed to support the EOM planning.

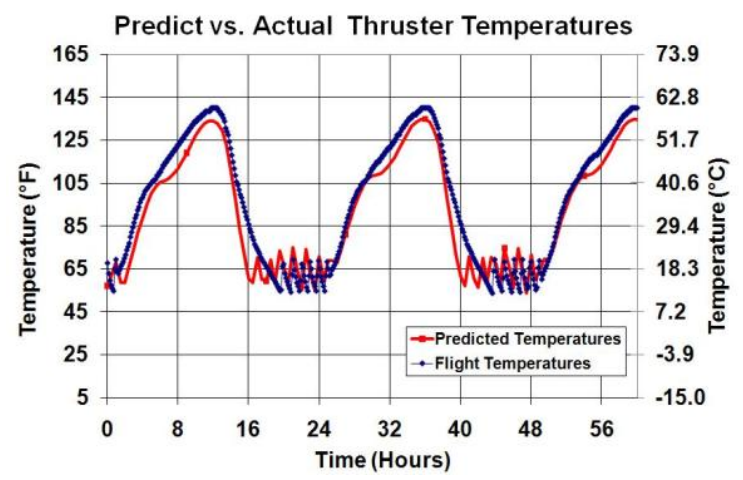

Figure 6 - Thruster P1A Temperature Correlation

The correlated TDRS-1 thermal model was then used to predict temperatures for the Z-spin configuration. The predicted Z-spin temperature results were used to determine whether any of the critical spacecraft component temperatures would violate qualification or acceptance limits. Figure 7 shows a sample temperature prediction for the spacecraft panel supporting critical communication electronics.

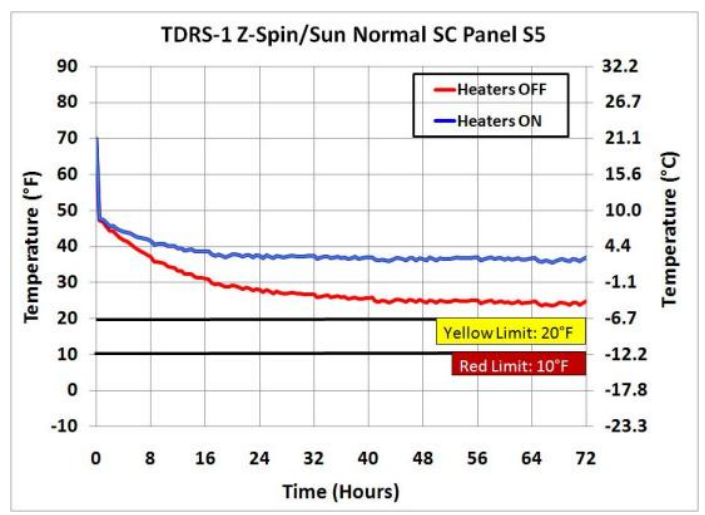

Figure 7 - Communication Panel Temperature Predictions

\footnotetext{
${ }^{8}$ Trade names and trademarks are used in this report for identification only. Their usage does not constitute an official endorsement, either expressed or implied by the National Aeronautics and Space Administration.
}

A joint effort between WSC and GSFC Sustaining Engineering teams to develop the spacecraft electronic and heater power tables (Table 1) was significant in establishing the TDRS-1 power dissipation assumptions used for thermal model temperature predictions.

Furthermore, this power dissipation data along with the thermal model predictions provided valuable insight into the heater power duty-cycle estimate that was critical in estimating the overall power balance during the Z-spin orientation.

Table 1 - TDRS-1 EOM

Z-spin power dissipation summary

\begin{tabular}{|l|r|r|}
\hline & $\begin{array}{r}\text { Primary Power } \\
(\mathrm{W})\end{array}$ & \multicolumn{1}{|c|}{$\begin{array}{c}\text { Total Power } \\
(\mathrm{W})\end{array}$} \\
\hline ATTITUDE CONTROL SUBSYSTEM & 17.19 & 17.19 \\
\hline Command \& Telemetry Units & 5.60 & 5.60 \\
ESE (Earth Sensor Electronics) & 15.20 & 15.20 \\
Solar Array Drive Assemblies & 75.00 & 75.00 \\
\hline$+X,-X$ Gyro Rate Asssembly (GRA) & SubTotal & 112.99 \\
\hline CONTROL ELECTRONICS SUBSYSTEM & 22.50 & 22.50 \\
\hline CEA - Control Electronics Assembly & SubTotal & 22.50 \\
\hline & & 9.30 \\
\hline ELECTRICAL POWER SUBSYSTEM & 9.30 & 1.70 \\
\hline PCU - Power Control Unit & 1.70 & 12.80 \\
DEA - Drive Electronics Assembly & 12.80 & 3.60 \\
SC Electronics Converter (SEC) & 3.60 & 27.40 \\
\hline Battery Scanner & SubTotal & \\
\hline & & 33.00 \\
\hline TELEMETRY TRACKING \& COMMAND & 33.00 & 23.50 \\
\hline TRANSPONDER & 23.50 & 56.50 \\
\hline CTU - Command \& Telemetry Unit & SubTotal & 24.70 \\
\hline COMMUNICATIONS SUBSYSTEM & & 24.70 \\
\hline Communication Converters & 24.70 & Total \\
\hline & & \\
\hline & SubTotal & \\
\hline
\end{tabular}

\section{Telemetry and Command Communication Issues}

TDRS-1 was equipped with a mast-mounted, omnidirectional, S-band antenna for use during launch and inorbit contingencies. This antenna has its bore sight offset $21^{\circ}$ from the Z-body axis in the Y-Z plane. An antenna pattern measured on a quarter-scale model during development ground testing showed the omni antenna pattern to be approximately $265^{\circ}$ in width, centered on the bore sight (Figure 8).

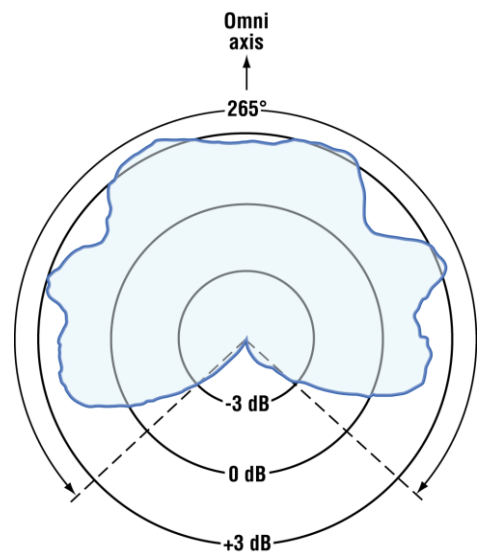

Figure 8 - Omni Antenna Coverage Pattern 
This pattern, coupled with the omni bore sight offset, meant that when a Z-spin is perfectly oriented to the orbit normal, communications via this antenna would be marginal once per revolution because the edge of the antenna null swept past the Earth. Analysis of the spacecraft dynamics during a modeled burn showed that a certain amount of nutation during thrusting was inevitable making it uncertain whether the T\&C coverage would be available to send the continuous stream of commands required for fuel depletion burns.

A continuous command stream was of particular importance since the dynamics during fuel depletion required continuous burns for an integral number of spacecraft rotations to avoid disrupting or tilting the orbit normal spin axis. Good windows of T\&C coverage following the burns were also important for providing the ranging data critical for accurate post-burn orbital solutions. Simulated spacecraft fuel depletion burn modeling provided insight, but true results would not be known until the omni antenna was utilized in the spinning attitude. To characterize the quality of the space-to-ground link through the antenna as well as provide real-time insight into disturbance of the orbit normal spin axis, it was planned to monitor the ground receiver's signal strength (Automatic Gain Control, AGC), on-board receiver signal strength, and telemetry dropouts.

It was thought that a 24-h plot of the on-board received signal strength and ground receiver AGC could be used to indicate the magnitude and plane of the Z-axis tilt with sufficient precision to allow for $\mathrm{Z}$-axis precession maneuvers to remove the tilt as needed. Patterns in the 24-h plot of on-board received signal strength and ground receiver AGC supported this belief but it was never validated since FDF thrust vector solutions provided sufficient insight for the Z-spin axis tilt adjustment.

\section{On-orbit Campaign and Performance RESULTS}

\section{Preparations and Organization}

Introduction-The early failure signature on the last operational SGL downlink TWT initiated the beginning of TDRS-1 EOM preparations. A TDRS-1 End-of-Mission Integrated Mission Plan (IMP) document [8] captured the diverse products that would be required to execute the EOM campaign. The IMP contained:

(1) EOM requirements, procedure objectives, and protocols.

(2) Organizational, management, and staffing structure.

(3) EOM ground resource management.

(4) EOM activity flowchart.

(5) Notional planning schedule.

(6) Executable Command Plans (nine sections).

(7) Flight rules.

(8) Contingency plans.
Flight Rules-Flight rules were created to establish predetermined decisions and operational boundaries which address safety, risk mitigation, and overall practicality based upon mutual agreements between NASA centers (WSC, GSFC, and JSC) to guide the conduct of TDRS-1 EOM activities. Flight rules were also mapped to the TDRS-1 EOM requirements in [3]. The Flight rules addressed:

(1) Orbit-raising: Defined OTH resources and alternatives for failure needed to reach the targeted GEO+350 kilometers.

(2) OTH Contingency Support: Allocated ground resources and limited types of OTH operations to orbit raising and passivation only.

(3) Fuel Depletion: Set limits on unintended perigee height-lowering, and fuel-depletion-time limits to OTH minus 3 days.

(4) Operating Time Constraints: Set goal to complete all requirements while in WSC station view. Since orbit raising left TDRS-1 in a non-geosynchronous orbit with a $4.6 \%$ drift rate, time to complete fuel depletion and equipment passivations, was limited.

(5) Isolation-Valve-Opening Constraints: Established constraints on operation of A-side propulsion system isolation valve to reduce the risk of resulting total impulse lowering perigee height

(6) Spacecraft Power Management: Established maximum battery depth-of-discharge limits, and minimum battery voltage.

(7) Thermal Management: Established EOM thermal limits for the remaining active spacecraft equipment.

(8) Equipment Passivation: Provided for passivation via alternative OTH stations.

(9) TDRS-1 EOM versus TDRS Fleet Contingency: Established response.

(10) Orbit Determination Requirements: Defined orbital determination fidelity and frequency for orbit-raising and fuel depletion.

(11) Ground Hardware and Software Management: Constrained hardware and software modifications during EOM for operational consistency.

(12) Flight-Rule Conflict Arbitration: Established a management protocol for flight-rule conflicts and exceptions.

Key Teams and Facilities - Two teams were used for TDRS 1 EOM.

The White Sands Complex team, located at White Sands, NM was composed of the EOM lead, flight directors, lead ACS, systems test conductors, a flight support team, and EOM systems engineers. The WSC team had two separately-located elements; one included the test conductor and his team of satellite controllers and systems/ACS engineers; the other was composed of flight dynamics and systems engineers. 
The GSFC team, located at Greenbelt, MD was composed of NASA Management, FDF (providing orbit determination after each maneuver), Conjunction Assessment groups (providing comprehensive orbital debris tracking), and collision assessment and discipline engineering expertise.

\section{Orbit-Raising-Summary of Results}

The initial estimate of orbit-raising burn durations did not take into account expected loss of station-keeping thruster-on-time as a consequence of attitude control thruster firings, which interrupted the station-keeping thrusters. Subsequent analysis [7] indicated that an additional $30 \mathrm{~s}$ would be required with each maneuver. Further analysis also indicated that the additional burn duration would present no significant increase in risk to the conduct of the maneuver. Thus, as executed, all twelve orbit-raising burns were $630 \mathrm{~s}$ in duration.

There was a gradual drop-off in achieved versus targeted drift rate over the 12 orbit-raising maneuvers. Starting with an underperformance of $1.05 \%$ for the first burn, the delta drift rate progressively declined to $6.31 \%$ for the last burn. Two key factors that contributed to the decline in achieved versus targeted drift rates were: first, the progressive increase in the negative yaw thruster duty-cycle for reasons related primarily to the increasing difficulty in controlling pre-maneuver roll/yaw momentum; and secondly, the gradual drop-off in station-keeping thruster force consequent to a gradual decline in propellant tank pressure.

In terms of the overall results (targeted versus achieved), the final drift rate was approximately $3.75 \%$ less than planned $(-4.54 \%$ versus $-4.72 \%$ d). The final perigee altitude (above GEO) was approximately $3.98 \%$ less than targeted $(351.72$ $\mathrm{km}$ versus $366.30 \mathrm{~km})$. Figure 9 shows the apogee and perigee history over the course of the orbit-raising burns through the fuel depletion burns.

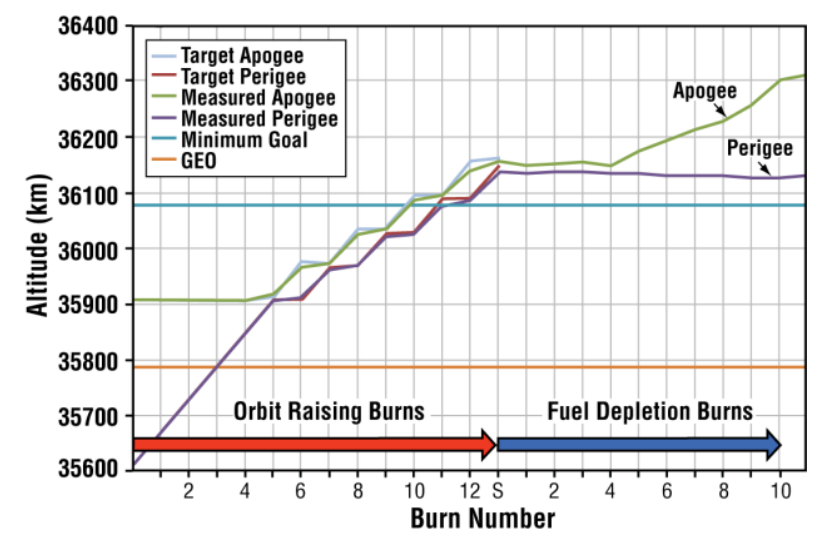

Figure 9 - TDRS-1 Altitude History for Orbit-raising and Fuel Depletion Burns

Propellant Summary-Based on propellant flow-rate calculations along with actual burn time, it was estimated that $144.88 \mathrm{~kg}$ of propellant was expended starting with orbit raising through spacecraft shutdown.
Accounting for the estimated residual propellant of $1.6 \mathrm{~kg}$ remaining in the tank and lines, the estimated propellant mass of $139.47 \mathrm{~kg}$ at the start of EOM was in error by 7.01 $\mathrm{kg}$.

Given that the total propellant mass, loaded prior to launch, was $597.83 \mathrm{~kg}$; the discrepancy in estimated remaining propellant mass works out to an error in propellant mass bookkeeping of approximately $1.2 \%$ over 27 years.

\section{Roll Maneuver and Z-Axis Spin-Up}

In order to point the spacecraft Z-axis to orbit normal, a positive roll maneuver was required. Upon completion of the orbit-raising $\Delta \mathrm{v}$ maneuvers and after settling into the normal Earth-pointing orientation, ground operators commanded the spacecraft into an inertial hold mode using gyroscopes and thrusters. The operators rolled the spacecraft $90^{\circ}$ by using a combination of the positive roll thruster and the yaw thrusters to accelerate and decelerate the roll rate. (There was a combination of yaw thrusters which had netzero yaw torque, but provided an effective negative roll torque.) The maneuver, practiced several times on a spacecraft simulator, was accomplished without significant incident.

Based on simulations, on-orbit maneuvers, and gyro accuracy, it was predicted that the Z-axis would be within $1^{\circ}$ of orbit normal following the roll maneuver. The $\mathrm{Z}$-axis was now pointed northward and normal to the orbit plane.

After a relatively short period which allowed the spacecraft to settle into its new orientation and an assessment of its state of health, the operators disabled all onboard attitude control modes and commanded a sequence of yaw thruster firings to spin-up the vehicle about the negative $\mathrm{Z}$-axis to approximately $1.0 \%$ s. Careful thought was given to the method used to spin the spacecraft up without introducing unnecessary (and not fully measurable) tilt away from orbit normal. The spin-up was performed in two steps: the first step was to spin the vehicle up to $0.5 \%$ s. There was a pause sufficient for the vehicle to complete one-half of a rotation about the Z-axis. Then the operators command the second step up in spin rate. It was shown by simulations during the planning phase that this two-step approach would leave a minimal residual nutation and would add, at most, $0.4^{\circ}$ to the offset from orbit normal. Spacecraft performance again matched simulation performance.

Nutation could not be directly measured, but could be inferred by recognizing that the transverse momentum that rotates in space due to nutation will cause the pitch and roll rates to cycle in quadrature. In fact, the magnitude of transverse momentum is constant and defined by the product of the peak pitch rate multiplied by the pitch inertia, which is equal to a similar calculation using roll rate and inertia. The nutation angle is defined by the ratio of the transverse momentum magnitude to the spin momentum. 
Thus monitoring the peak pitch and roll rates is equivalent to monitoring the nutation angle.

After completing the spin-up, the operators observed a very minor nutation $\left(\sim 0.6^{\circ}\right.$ with an 8 -min period $)$ from the gyro roll and pitch rates (Figure 10a) thereby validating the twostep spin-up approach suggested by the analytic simulation. No further action was necessary.

\section{Torque-Free Rotational Dynamics}

During the nearly 20-hour post spin-up observation period, the spacecraft rotation established about the Z-axis was stable and unchanging, as was predicted by analysis and simulation. This was established by monitoring two parameters: the spin rate of rotation and the nutation angle. The first parameter was measured directly using data from the spacecraft gyros. It did not change over the observation period.

The second parameter, nutation angle, was inferred from the gyro data as discussed earlier. A comparison of the derived rate plots taken at the end of the spin-up and prior to the first fuel depletion burn (Figures 10a and 10b) indicated that the nutation angle decreased by approximately $6 \%$ over the observation period. This can be explained by spacecraft structural- or fuel-damping.

\section{Power Management On-Orbit Performance}

During the planned 20-hour period allocated for evaluation of subsystem performance, it became obvious that the solar array voltage and current output was well in excess of that necessary to maintain battery state-of-charge during the Z-spin. In addition, the solar heating resulting from the flat spin kept all spacecraft surfaces sufficiently warm, such that no heater loads were required.

However, a potentially damaging overcharge situation now had to be addressed. To avoid battery damage, predetermined loads that were originally shed in anticipation of a marginal power condition were progressively turned back on to stabilize battery charging to a safe level.

Unfortunately, the algorithm that was developed to help support battery management by tracking battery charge/discharge balance was unable to accurately integrate the charge and discharge current due the 32-s telemetry sample rate combined with a 6-min spacecraft rotation rate. This reduced the accuracy of the calculations and also resulted in an aliasing effect due to the periodicity of the data. Battery voltage and individual cell voltages were used as state-of-charge indicators instead of using the originally planned charge-discharge ratio in combination with the composite of cell voltages. Benign spacecraft loads were cycled as necessary in order to maintain stable and safe battery voltages. Cell voltages on Battery 3 began diverging somewhat high after several days into fuel depletion, indicating slight overcharge, but all 24 cells remained within an acceptable range. As a precaution against excessive battery overcharge to Battery 3 during the final shutdown sequence (when loads have to be turned off), the shutdown sequence was altered to end with Battery 1 (the lowest stateof-charge battery) on-line instead of the planned Battery 3 .

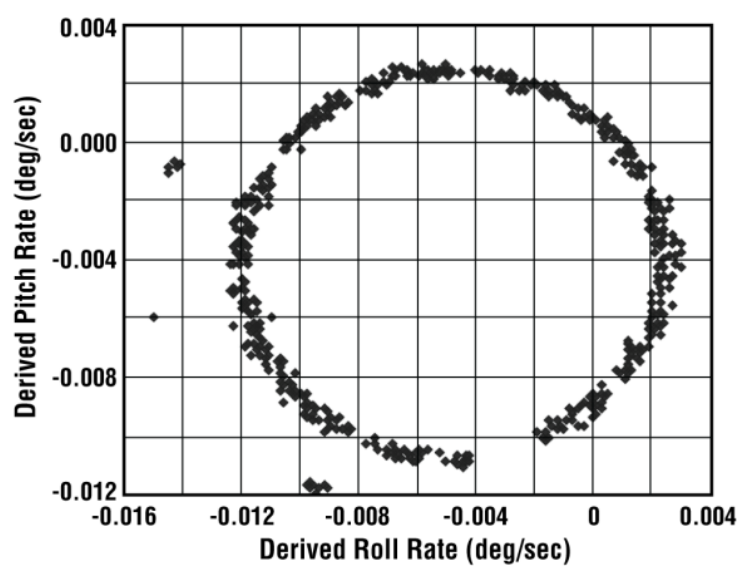

Figure 10a - Nutation Cycle After Spin-up

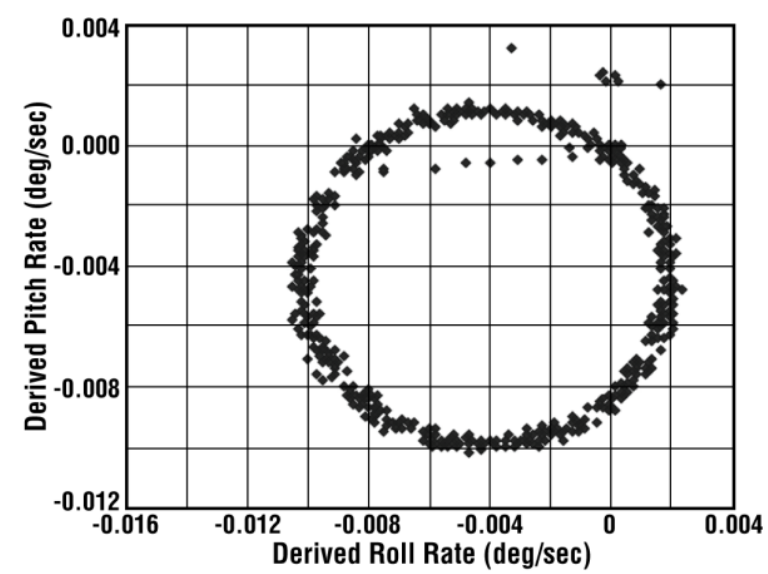

Figure 10b - Nutation Cycle Prior to First Fuel Depletion Burn

Fortunately, the net power balance (one of the most potentially complicating conditions to the primary fuel depletion approach) was easily managed. It is worth noting that the extensive preparation made by identifying, classifying, and quantifying loads to achieve minimum power proved to be time well spent, as it served as the basis for incrementally applying loads to control charge.

\section{Thermal Subsystem On-Orbit Performance}

The TDRS-1 TMM proved to be a valuable tool for predicting temperatures and heater power estimates for different TDRS-1 electronic power, heater power and orbit configurations. The TDRS-1 thermal subsystem performance throughout the EOM phase was as predicted. Figure 11 shows the very close match between the solar array's predicted versus actual temperatures for the Z-spin. 
The Z-spin orbit normal flight temperatures obtained during the TDRS-1 EOM also verified that critical components (i.e., transponder, RCS, EPS, and batteries) for the TRWseries TDRS spacecraft can be maintained above qualification limits and utilize minimum heater power when in this orientation.

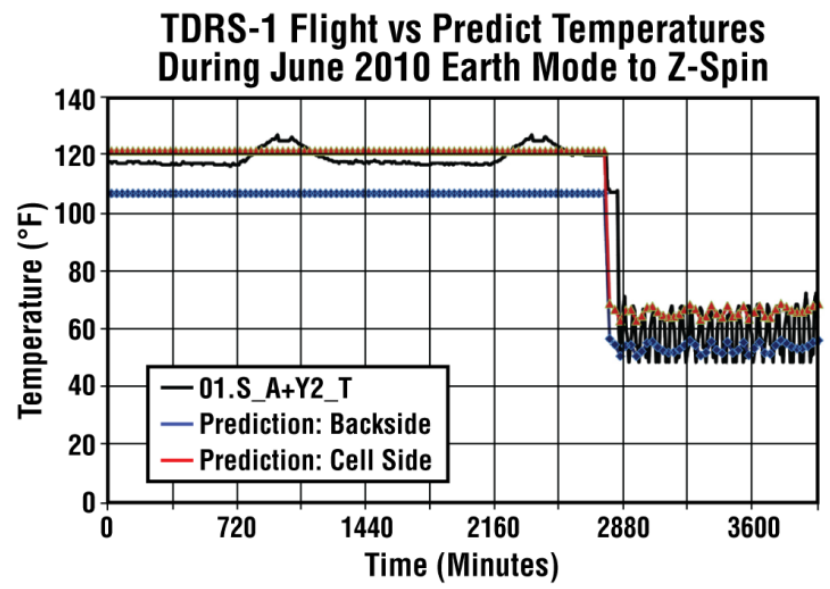

Figure 11-Solar Array Z-spin Temperature Comparison

\section{Telemetry and Command On-Orbit Performance}

The first four fuel depletion burns were relatively $\mathrm{T} \& \mathrm{C}$ dropout-free with the Z-axis oriented within $4^{\circ}$ of orbit normal according to FDF thrust vector solutions. From Burn-5 through the completion of fuel depletion burns, T\&C became increasingly intermittent because of the growing Z-axis tilt-which approached $8^{\circ}$. The telemetry signal-strength pattern followed a superposition of the spacecraft's rotation, nutation, and precession originating from the fuel depletion attitude perturbations (Figure 12).
As fuel depletion proceeded, the on-board received signal strength and telemetry dropout patterns were trended. This data indicated that as the $\mathrm{Z}$-axis tilt increased, a combination of the SGL antenna feed and C-band antenna feed occulted the omni resulting in an increased number of $\mathrm{T} \& \mathrm{C}$ dropouts. For the most part, the dropouts were 1 or $2 \mathrm{~s}$ long with occasional dropouts on the order of $10 \mathrm{~s}$. None of the dropouts impacted burns. The C-band feed is fixed (residing on the same boom as the omni), but the SGL antenna resided on a gimbaled arm that was parked slightly above the spacecraft body/face. The SGL antenna was lowered by nearly $10^{\circ}$ (approximately $0.40 \mathrm{~m}$ ) with the thought that it could only improve T\&C. After the change, the overall the number and duration of dropouts remained essentially unchanged.

The team concluded that the C-band antenna feeds (in combination with $\mathrm{Z}$-axis tilt) were the primary dropout contributors. With sufficiently tight management of the nutation angle, the tilt of the angular momentum from orbit normal, and an ability to take advantage of the good daily communication periods, T\&C dropouts did not interrupt any of the fuel depletion burns and had little impact to the mission.

\section{Fuel Depletion Burns On-orbit Performance}

A series of 10 fuel depletion burns were executed as part of the TDRS-1 EOM campaign. Table 2 summarizes the burn parameters. All but one of the fuel depletion burns were executed near perigee to minimize the impact of the burn upon perigee altitude. Previous analysis had determined that perigee must remain $290 \mathrm{~km}$ above GEO to meet orbital debris requirements. The maximum perigee decrease for a burn was $3.5 \mathrm{~km}$ resulting from Burn-5.

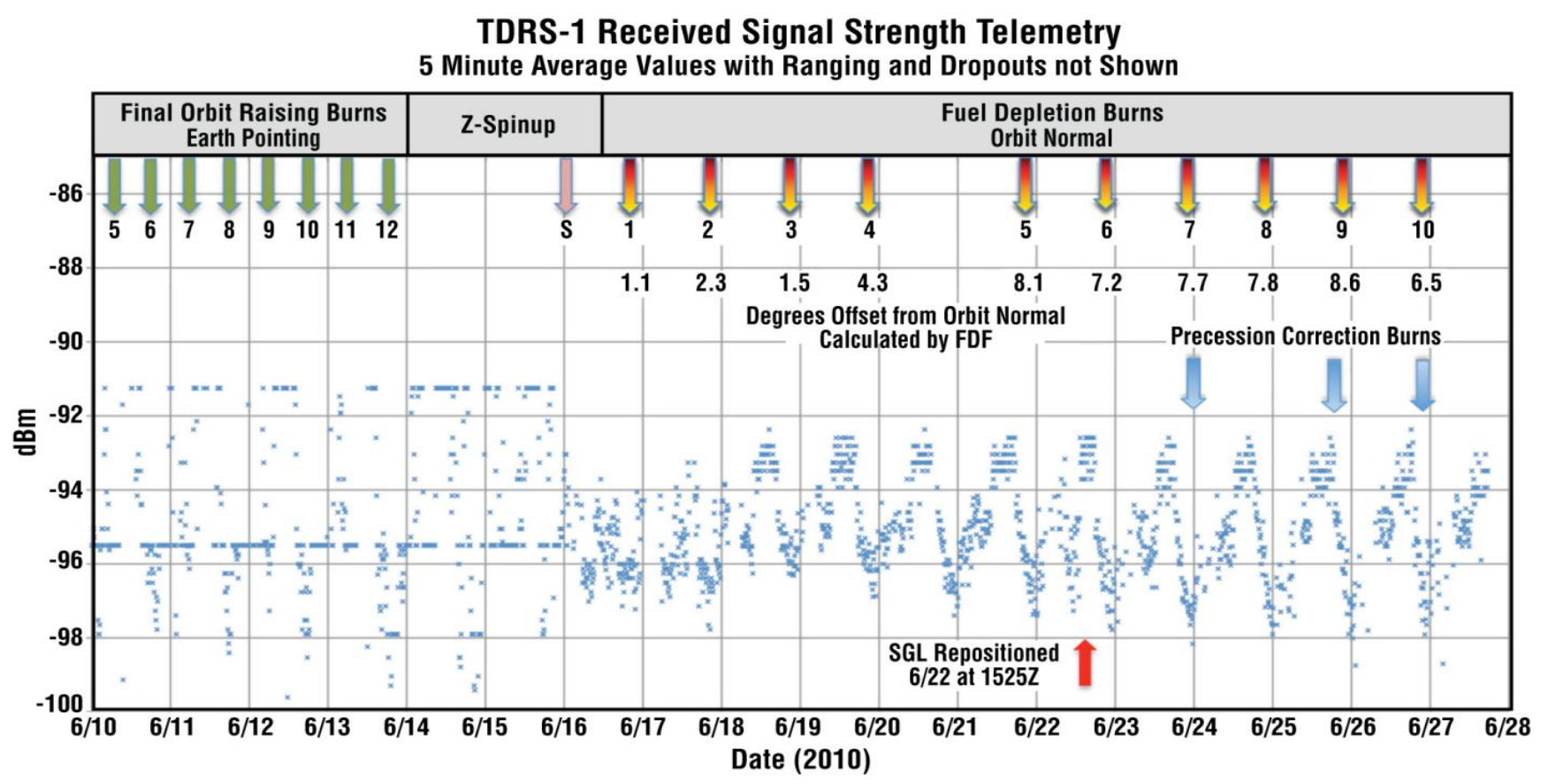

Figure 12 - Received Signal Strength Telemetry 
Table 2 - Summary of Fuel Depletion Burns

\begin{tabular}{|c|c|c|c|c|c|c|c|c|}
\hline $\begin{array}{c}\text { Burn } \\
\text { no. }\end{array}$ & DOY & $\begin{array}{l}\text { Burn start orbit } \\
\text { position } \\
\text { (h/min before perigee) }\end{array}$ & Duration & $\begin{array}{c}\text { Inertial } \Delta v \\
\text { magnitude } \\
(\mathrm{m} / \mathrm{s})\end{array}$ & $\begin{array}{c}\text { Inertial } \Delta v \\
\text { angle from } \\
\text { orbit normal } \\
\text { ( })\end{array}$ & $\begin{array}{c}\text { Inertial } \Delta v \\
\text { angle from sun } \\
\text { vector } \\
\left(^{\circ}\right)\end{array}$ & $\begin{array}{c}\Delta \text { Apogee } \\
(\mathrm{km})\end{array}$ & $\begin{array}{c}\Delta \text { Perigee } \\
\text { (km) }\end{array}$ \\
\hline 1 & 167 & $32 \mathrm{~m}$ & $1 \mathrm{~h} 9 \mathrm{~m} 0 \mathrm{~s}$ & 1.6 & 1.1 & 157 & 1.9 & 1.7 \\
\hline 2 & 168 & $\begin{array}{l}\text { Burn centered on } \\
\text { descending node }\end{array}$ & $1 \mathrm{~h} 10 \mathrm{~m} \mathrm{46s}$ & 1.7 & 2.3 & 143.7 & 3.6 & 0.4 \\
\hline 3 & 169 & $1 \mathrm{~h} 4 \mathrm{~m}$ & $1 \mathrm{~h} 53 \mathrm{~m} \mathrm{17s}$ & 3.4 & 1.5 & 122.8 & 5.1 & -0.9 \\
\hline 4 & 170 & $58.5 \mathrm{~m}$ & $3 \mathrm{~h} 6 \mathrm{~m} 7 \mathrm{~s}$ & 3.7 & 4.3 & 136.6 & 16.8 & -2.1 \\
\hline 5 & 172 & $18 \mathrm{~m}$ & $3 \mathrm{~h} 3 \mathrm{~m} 0 \mathrm{~s}$ & 4.6 & 8.1 & 82.4 & 16 & -3.5 \\
\hline 6 & 173 & $1 \mathrm{~h} 22 \mathrm{~m}$ & $2 \mathrm{~h} 2 \mathrm{~m} 47 \mathrm{~s}$ & 2.9 & 7.2 & 81.2 & 18 & -1 \\
\hline 7 & 174 & $1 \mathrm{~h} 25 \mathrm{~m}$ & $1 \mathrm{~h} 38 \mathrm{~m} 54 \mathrm{~s}$ & 2.2 & 7.7 & 90.6 & 18 & -1 \\
\hline 8 & 175 & $2 \mathrm{~h} 11 \mathrm{~m}$ & $3 \mathrm{~h} 20 \mathrm{~m} 58 \mathrm{~s}$ & 4.1 & 7.8 & 92 & 32 & -1.5 \\
\hline 9 & 176 & $2 \mathrm{~h} 21 \mathrm{~m}$ & $4 \mathrm{~h} 24 \mathrm{~m}$ & 5.2 & 8.6 & 104.2 & 23 & -0.8 \\
\hline 10 & 177 & $3 \mathrm{~h} 41 \mathrm{~m}$ & 1h $46 \mathrm{~m} 58 \mathrm{~s}$ & 2.1 & 6.5 & 93 & 10.5 & 0.4 \\
\hline
\end{tabular}

*This angle is measured in the orbit plane.

The final orbit achieved was 341 by $531 \mathrm{~km}$ above GEO and satisfied the prescribed EOM altitude requirements. The burn durations were determined by the time required to complete a pre-determined number of spacecraft revolutions and followed a gradual build up from a nominal 72 minutes to near the $3 \mathrm{hr}$ qualification limit. This allowed confidence to be gained and enabled control of the post-burn position of the angular momentum vector. The amount of time required to complete revolutions varied with the actual spin rate, which itself varied during the burns.

Table 2 also shows the magnitude and orientation of the inertial $\Delta v$ resulting from the burns. These parameters were obtained as a product of the orbit determination process. Some inertial $\Delta \mathrm{v}$ is observed because the thrusters had a component of their thrust aligned with the $\mathrm{Z}$-axis which was not cancelled by the spin. In addition, spacecraft nutation can result in variation of thruster inertial pointing. As designed, the inertial $\Delta \mathrm{v}$ realized during the Z-axis normal spin-mode was well below the approximately $155 \mathrm{~m} / \mathrm{s}$ available, given the amount of propellant expended and the thruster's specific impulse. The $\Delta \mathrm{v}$ angle from orbit normal was controlled throughout the burns to less than $9^{\circ}$ offset. This was accomplished by controlling burn stop-time, the nutation angle, and by precession maneuvers to realign the angular momentum closer to orbit normal. A more complete discussion of these techniques follows.

\section{Post-burn Nutation Management}

Ground operators were generally successful in ending the fuel depletion burns at nearly the ideal time that would minimize the residual nutation, which continued in the torque-free situation that followed. Nonetheless, each burn did end with some small nutation, which was minimized before the next burn. The classical technique for damping nutation is to fire a thruster that provides a torque normal to the momentum vector. A pitch thruster was selected for use on TDRS1 because both polarities were available (versus constraint on using only the positive roll thruster).

As discussed earlier, firing the thrusters while the spacecraft is rotating will cause both nutation and precession. Simulations found that firing the positive pitch thruster in three equal sets of burn-times - each spaced by one nutation period-were successful in reducing nutation without precessing the angular momentum vector. This technique was utilized at the end of each fuel depletion burn (Figure 13).

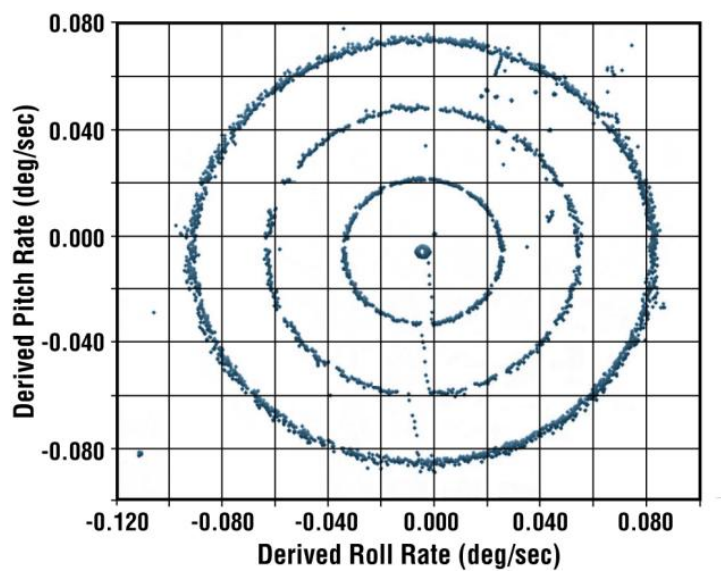

Figure 13 - Example of Nutation Damping in Three Pitch-thruster Firings to Minimize Precession Inertial Motion of Z-axis and Angular Momentum Vector 
Minimizing the Z-axis offset from orbit normal was desirable so that the thrust components along the $\mathrm{Z}$-axis (the cant of the $\mathrm{Z}$ thruster pairs add a velocity component, $\sin 17^{\circ}$, in the $+Z$ body direction) would not adversely affect the orbit and $\mathrm{T} \& \mathrm{C}$ coverage. Applied along the orbit normal, itself, these components would only serve to raise or lower the orbital inclination which did not impact requirement compliance. The Z-spin characterization period following initial spin-up reinforced the assumption that the Z-axis was some small angle from orbit normal as the T\&C link quality was consistent around the orbit and coarse sun sensors (CSS) readings agreed with beta angle values.

Fuel Depletion Burns - The basic strategy for executing the burns while maintaining near orbit normal pointing relied upon understanding the path that the angular momentum vector would follow while the spacecraft was thrusting. Simulations had shown, see Figure $4 \mathrm{~b}$, the angular momentum would follow a circular path in inertial space returning near to its origin (position at burn start) every spin period. By restraining the burn durations to be integral numbers of spin periods (and nutation periods to bring the Z-axis back near the origin as well) net angular momentum vector travel resulting from the burn was minimized. The nutation and spin periods were approximately a 4:3 ratio with a least-common-period of approximately $24 \mathrm{~min}$ (four spin periods).

Angular momentum vector movement relative to the orbit normal was minimized but not eliminated through the execution of the series of burns due to achievable accuracy in terminating the burns and differences in individual thruster performance. On orbit characterization of the two thrusters used, Z1 and Z2 had already revealed a thrust performance difference of approximately $3 \%$.

Fortunately a disturbance torque compensation (DTC) feature of the spacecraft allowed for a simple memory load to effect off-modulation of the higher performing thruster. It was observed during the very first fuel depletion burn that adjustments to the DTC were required to maintain the spin rate near its nominal $1 \%$ value. As this pattern of spin rate variation continued over the next few burns it became apparent individual thruster performance was changing during the burns enough to affect the dynamics. The most noticeable single event occurred at the beginning of Burn-4 when the spin rate changed by $0.017^{\circ} /$ s over a $19-\mathrm{s}$ period. While on-orbit execution of the fuel depletion burns was subject to this non-simulated effect, it is worth noting the on-orbit inertial motion of the Z-body unit vector generally followed the path predicted by the dynamic simulations. Compare the simulation (Figure 4b) with the flight data (Figures 14a-d), which were produced by processing TDRS1 gyro outputs [9]. The same can be noted for the angular momentum path, inferred in Figure 14a by connecting the cusps of the Z-body path. A great deal of the success of the fuel depletion burns is due to the accuracy of the dynamic simulations in predicting the behavior of the spacecraft rotational dynamics.
The variations in individual thruster performance served to walk the angular momentum vector around in inertial space in an unpredictable manner contributing to the variations of the $\Delta \mathrm{v}$ from orbit normal shown in Table 2.

\section{Burn Planning and Evaluation}

The initial two fuel depletion burns were planned for nominal durations of $72 \mathrm{~min}$. A conservative approach was taken for Burn-1, which centered the burn about perigee to minimize any potential burn impacts to perigee height. This provided a "coarse" burn start time of perigee passage minus $36 \mathrm{~min}$ (the "fine" burn start time also takes into account the spin phase). An observation made from the dynamic simulations - discussed previously - found that the angular momentum vector will follow a repetitive circular path during the burn that is biased off from the origin in a predictable direction. The net effect would be to realize a tilt from the origin of the $\Delta v$ applied equal to the angular radius of the angular momentum path; the Z-body axis is also rotating about this point but in a much more complex path. It was desirable to align the $\Delta \mathrm{v}$ tilt in the same direction as the spacecraft's velocity vector so as to increase orbital energy, and apogee height, rather than the opposite. An inertial reference point was needed during a spin period to precisely determine the "fine" burn start time such that the correct tilt of the $\Delta v$ would be achieved. The spacecraft's CSSs were capable of providing just such a reference. The CSSs were placed on the outer corners of the solar arrays and designed to provide pitch and yaw inputs for the spacecraft's Sun mode. However because of the Z-spin orientation, the CSS's yaw reading crossed through zero as the array normal pointed in the Sun direction. By defining a phase angle offset from the CSS yaw ascending zero crossing and starting the burn at that point in the spin, the proper orientation of the $\Delta \mathrm{v}$ tilt was achieved. The initial results were encouraging as both apogee and perigee heights were increased.

Burn-2 was centered on the descending node. Poor T\&C link quality had been observed during the first burn, and it was thought the descending node might provide a better space-to-ground geometry. Burn-2's T\&C link quality did not noticeably improve so subsequent burns returned to the near-perigee strategy. Burn-2's $\Delta \mathrm{v}$ tilt was again properly aligned increasing both apogee and perigee. Since the angle from orbit normal had grown to $2.3^{\circ}$, a decision was made to align the tilt of the $\Delta \mathrm{v}$ opposite to the spacecraft velocity vector for Burn-3 in expectation of reducing the angle from orbit normal, and to accept some potential lowering of perigee. This strategy proved successful as the angle from orbit normal was reduced to $1.5^{\circ}$ while only lowering perigee by $900 \mathrm{~m}$. Burn-4, the longest planned to date at $192 \mathrm{~min}$, returned to the nominal strategy of aligning the $\Delta \mathrm{v}$ tilt in the same direction as the spacecraft's velocity approximately centered around perigee. This burn resulted in a surprising increase in the angle from orbit normal to $4.3^{\circ}$. 


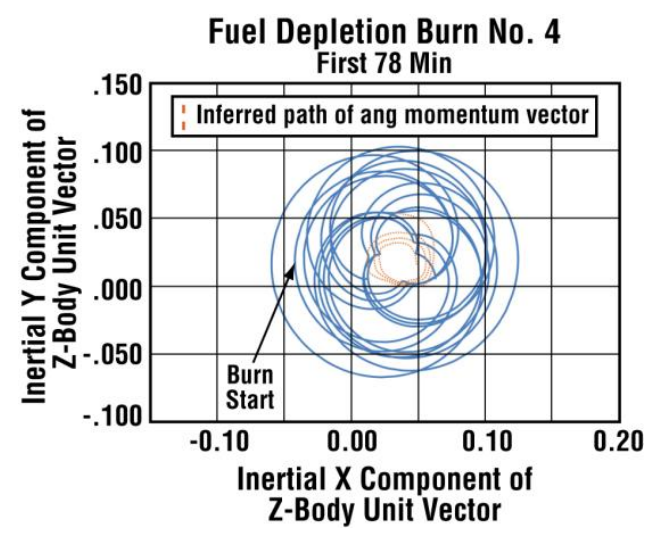

Figure 14a - Z-axis and Angular Momentum Vector Trajectories from Burn Start

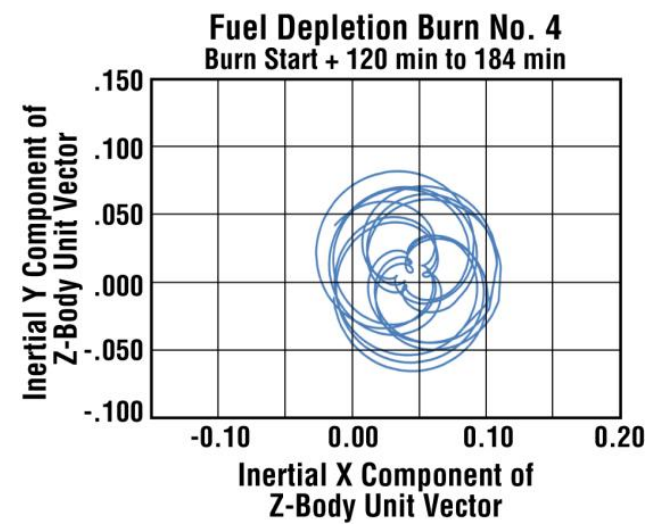

Figure 14c - Z-axis Trajectory from Burn Start +120 min to 184 min

Subsequent analysis has shown the angular momentum vector motion had wandered from its predicted path due to an initial thruster performance transient and continuing thruster variations during the course of the burn (Figures 14a through 14d). Burn-5 returned to the strategy implemented for Burn-3 with the intent of reducing the $4.3^{\circ}$ tilt from orbit normal. However, the strategy proved unsuccessful as the angle from orbit normal was increased to $8.1^{\circ}$. It is suspected that thruster variations once again had allowed the angular momentum vector to follow an erratic path.

Both Burn-6 and Burn-7 were executed with the nominal strategy used for Burn-2 and Burn-4. While the angle from orbit normal remained above $7^{\circ}$, the changes to apogee and perigee were acceptable. The final three burns $(8,9$, and 10) retained the nominal strategy but were preceded by precession maneuvers that sought to reduce the angular momentum vectors tilt from orbit normal. There was concern that final passivation could be impacted by a large tilt angle, so precession was used to mitigate this risk. The final reported angle from orbit normal was an acceptable $6.5^{\circ}$.

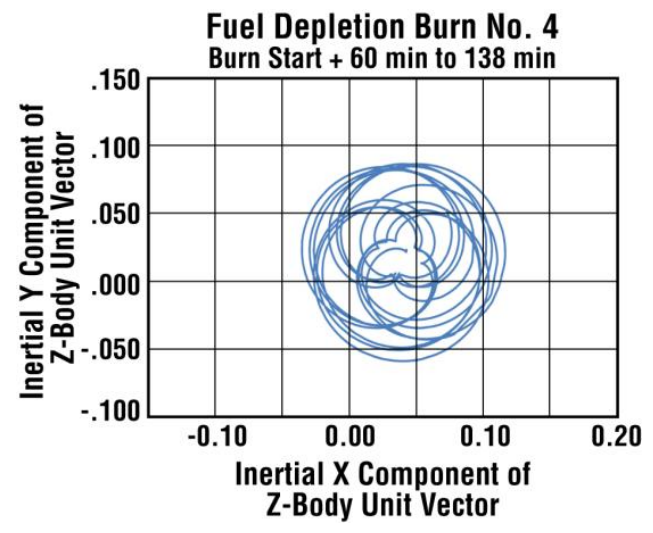

Figure 14 b - Z-axis Trajectory from Burn Start $+60 \mathrm{~min}$ to $138 \mathrm{~min}$

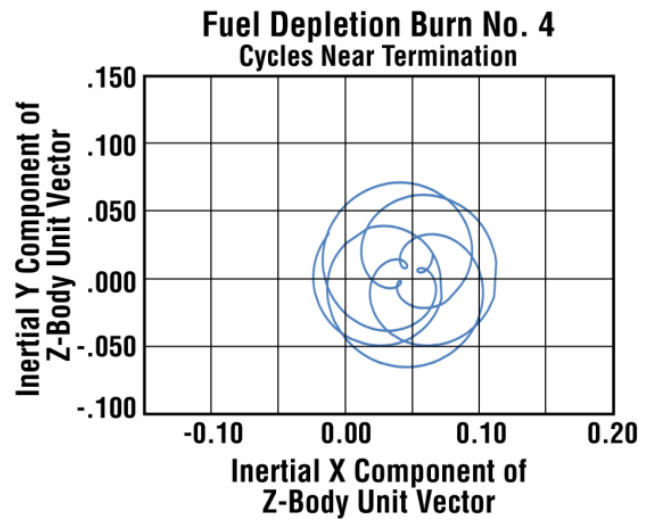

Figure 14d - Z-axis Trajectory Near Burn Termination

\section{FDF Support of Fuel Depletion Burns}

The GSFC FDF provided predictive spacecraft acquisition data before each burn and a post-burn spacecraft state and a best-fit impulsive $\Delta \mathrm{v}$ following each burn.

Orbit Determination-The post-burn state was determined using the Goddard Trajectory Determination System, a batch least-squares orbit determination program. About six hours of tracking was used for each solution, including T\&C range measurements from WSC and range/Doppler measurements from other ground stations.

Maneuver Modeling - It was assumed that the fuel depletion maneuvers could be modeled as an impulsive $\Delta \mathrm{v}$. The maneuvers were long enough to be noticeably nonimpulsive, but the resulting error was found acceptable. It was further assumed that the impulsive $\Delta \mathrm{v}$ would be the same for consecutive maneuvers. Though the maneuver direction did vary noticeably, the variation was sufficiently small between maneuvers for these assumptions to be useful in planning burns. The best-fit impulsive $\Delta \mathrm{v}$ for each burn was determined by minimizing the total position difference between a definitive post-maneuver ephemeris and premaneuver vector with the delta-V applied. 


\section{Z-Axis Precession Maneuvers}

Through the course of the fuel depletion burns, it became evident from the orbit determination trends that the orientation of the Z-axis was drifting.

During the burns, variations in individual thruster performance were causing the angular momentum to precess away from its position at burn start along an irregular path that did not return to the starting position. This was also evident in the effective orientation of the thrust vectorinertial $\Delta v$ angle from orbit normal-at the subsequent burns. See Table 2. As the tilt became larger, a decision was made to precess the Z-axis back toward orbit normal. The following discusses the two parts of the planning for precession maneuvers: the technique and the spatial timing.

Technique-As discussed above, external torques acting on a rotating body will affect spin speed, nutation, and precession of the spin axis. Just as a technique was found that adjusts nutation without affecting precession, what was needed here was the converse. Again using the analytic simulation, it was found that spacing a pair of thruster firings two spin periods apart (i.e., two rotations) - as opposed to one nutation period for nutation control-would result in a desired change in precession, but leave minimal residual nutation.

Spatial Timing-One of the outputs from the orbit determination activity was to place the effective $\Delta \mathrm{V}$ direction, and by inference the Z-axis tilt, in inertial space referenced to the Sun direction. Again, the decision was made to use the positive pitch thruster to provide the torque to precess the Z-axis. The next problem was to determine when the pitch axis was going to align with the spin tilt. The same technique that was used to determine burn start time within a spin period was applied. Given that there is no direct measurement of the orientation of the spin axis in space, there is the knowledge of when the solar arrays are pointed at the Sun, which can be a useful reference point.

With excellent knowledge of the spin rate, the spatial orientation of the body axes can be inferred at any time relative to those Sun crossings. The procedure was to then issue thruster commands at approximately the time following the Sun crossing when the positive pitch thruster was opposed to the Z-axis tilt.

There were three precession maneuvers. The first two provided a small adjustment to validate the technique. The last one was more aggressive in righting the spin axis and consisted of three sets of back-to-back precession commands. While there were no direct observables of the angular momentum precession, Figures 15a and 15b shows plots of actual spacecraft derived roll and pitch rates during the last precession adjustment.

Figure 15a shows the initial small nutation; then an increase of nutation after the first firing of a pitch thruster (when the spin phase is such that the pitch thruster is aligned for the desired precession), and then a reduction in the nutation following the second thruster firing when the roll rate was near zero. This was repeated for a total of three firing pairs.

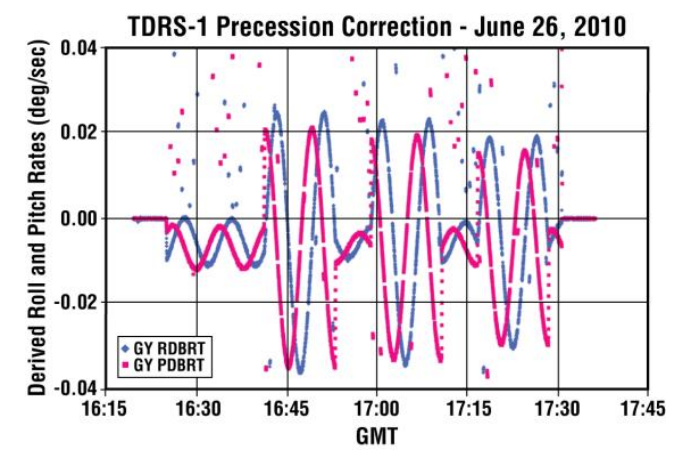

\section{Figure 15a - Derived Body Rates During Precession Adjustment}

Figure $15 \mathrm{~b}$ shows a rate plot for the same data. The figures show the effectiveness of the technique in leaving the system with very low nutation. The reduction in reported $\Delta \mathrm{v}$ tilt in the succeeding fuel depletion burn confirmed that the maneuver achieved the desired effect.

TDRS-1 Precession Correction June 26, 2010

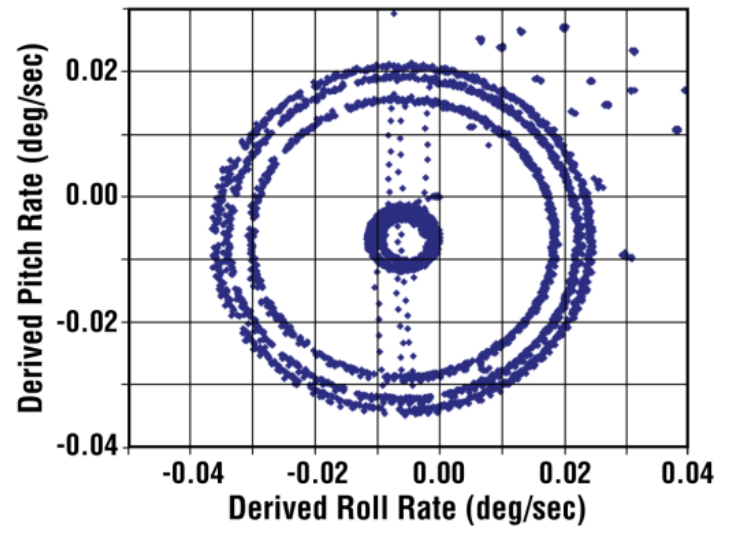

Figure 15b - Body Rates During Precession Adjustment

\section{Tank Depletion}

An assumption that the quantity of hydrazine decomposition gases trapped under the TDRS F-1 fuel tank diaphragms was small proved to be correct and the pressure was blown down to 100 psia without first depleting hydrazine. Figure 16 shows the hydrazine-side pressure signature as the two tanks began to deplete.

A rapid drop in the tank pressure is the signature of fuel tank depletion. Each of the two fuel tanks was a 1-m diameter ellipsoid with a flexible rubber radial ribbed diaphragm separating the nitrogen pressurant from the fuel. As fuel is removed from the tank, less liquid is available to displace the diaphragm, and the diaphragm moves closer to 
the tank walls. Near the point of total fuel depletion, the radial ribs of the diaphragm eventually come in contact with the tank wall. Some additional diaphragm displacement occurs between the ribs after contact, at which point the rapid fuel-side pressure drop is observed. Based on tank geometry, diaphragm rib height and rib spacing, residual fuel remaining (for both tanks combined) is calculated to be $0.6 \mathrm{~kg}$. Another $1 \mathrm{~kg}$ was calculated to remain in the fuel lines, (based on fuel line geometry).

The TDRS-1 design provided no means of venting the gaseous nitrogen from the pressurant-side of the tanks; they will remain pressurized at about 130 psia on the pressurantside (even though the liquid-side dropped to $106 \mathrm{psia})$. The residual $\sim 1.6 \mathrm{~kg}$ of fuel at the completion of the fuel depletion phase was considered negligible.

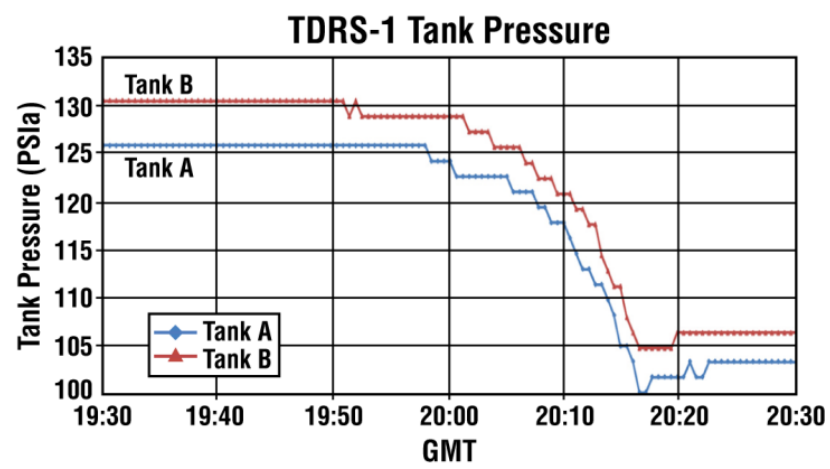

Figure 16 - Tank Pressure Signatures at Depletion

Fuel depletion was deemed successful and compliant with the orbital debris requirements by the NASA Orbital Debris Program Office.

\section{Passivation}

Equipment "passivation" or deactivation was driven by the EOM requirement to deactivate all spacecraft equipment that contained or produced mechanical (kinetic), chemical/explosive, RF, or electrical energy. Given the systems interdependency, careful consideration was given to the order of equipment passivation to avoid deactivating a component upon which other needed systems relied such as power converters, heaters, command and telemetry boxes, etc. TDRS-1 passivation was divided into three general phases as follows:

Phase 1-When TDRS-1 could no longer support user operations, a subset of payload equipment was turned off. The approach was to deactivate as much un-needed equipment as possible, as early as possible beginning with the payload equipment. This was done in such a way as to gather thermal and power modeling data, which spread the passivation sequence over several days for payload. Equipment passivation began with the deactivation of the SSA and MA Forward and Return service components on 21 October 2009. TWT \#1 was left on for failure trending and was finally deactivated 11 January 2010. The remaining payload equipment deactivation required EOM plan approval.

Phase 2-Equipment passivation-beyond what is normally done to place a spacecraft in temporary on-orbit storagewas prohibited prior to EOM plan approval to ensure that no irreversible passivation actions were taken. In March 2010, NASA Headquarters approval was granted to continue with deactivation. The remaining payload was deactivated in thermally-paced phases over 5 days starting on 17 March 2010. Deactivation was attempted on the SAC heaters on 25 March, but concern was raised that the SA antenna gimbal drives which were needed for momentum control during orbit raising might get too cold. This action was deferred and the SAC heaters were finally deactivated 16 June 2010 immediately following the final orbit-raising burn. Upon completion of Phase 2, only equipment required for the fuel depletion burns remained activated.

Phase 3-Phase 3 was divided into three parts in the mission plan [8]. The emphasis was placed on completing Phase 3 as quickly as possible, since critical spacecraft functionality was progressively disabled.

The first part of Phase 3 consisted of the deactivation of all ACS and propulsion equipment. The Z-spin configuration was ideally suited to support this deactivation because it used no on board ACS control laws or thruster firings. It totally relied upon the stiffness produced by the rotation about the Z-axis for inertial attitude stability. After ACS deactivation, spacecraft attitude telemetry was no longer available.

The second part of Phase 3 consisted of an initial EPS and final thermal passivation, which included disabling all remaining spacecraft main body heaters, and disconnecting Batteries 2 and 3-leaving only Battery 1 for load support. Batteries were passivated by disconnecting both the full and trickle charge solar array strings, and by connecting reconditioning resistors to the battery terminals to deplete the remaining battery charge. The TDRS design had no solar array/main bus disconnect function.

The third part of Phase 3 consisted of the final EPS and tracking, telemetry and command (TT\&C) deactivations. Due to the Z-spin configuration, the solar arrays rotated from Sun facing to anti-Sun facing and back over a 12 min period. This presented an approximate 5.5-min window where the solar arrays could power the bus and allow any final commanding. During this window, the last battery was disconnected and passivated. The final TT\&C passivation sequence began by turning off the transmitter power amplifier (PA) (resulting in termination of spacecraft telemetry downlink) and verifying carrier drop on the spectrum analyzer. This was immediately followed by a final command system reconfiguration sequence. Phase 3 was executed on 27 June 2010 over a 128-min period, which brought TDRS-1 
EOM activities to a close. All system passivations were verified as successful either directly in telemetry, by spectrum analyzer, or via multiple failed attempts to reacquire the spacecraft transponder.

\section{CONCLUSiOnS}

Several conclusions can be reached as a result of the TDRS1 EOM campaign spanning programmatic to subsystem level subjects.

\section{Programmatic}

Two factors determined the success of the EOM campaign and only one of the two was controllable. The factor not under EOM team control was failure of any critical nonredundant spacecraft equipment. This factor can only be controlled prior to the point where a failure compromises the spacecraft. Although TDRS-1 experienced an early orbit loss of redundancy, fortunately no critically disabling failures ensued, which allowed its operational mission and EOM to successfully be completed. The factor that was under EOM team control was thoughtful preparation and careful execution of the EOM plan. This methodology is consistent with NASA and corporate project management guidelines, but some key specifics are worth noting for the benefit of future EOM teams.

(1) Assemble the most experienced engineering team available to plan and implement the EOM activity. EOM planning for spacecraft such as TDRS-1, with numerous problems or failures, should obtain the support from key team members who helped resolve the original EOM-related critical spacecraft anomalies.

(2) Involve all teams early in the planning process. Understand all team member concerns and system constraints.

(3) Define "flight rules" to anticipate issues that may be encountered. Set decision criteria during the EOM development process - do not wait until the execution phase when prompt decisions may be required. The flight rule concept also facilitates the process of seeking pre-approval for risky and/or irreversible actions.

\section{System}

At the system level, the foremost lesson to be learned is that an outside-the-box concept not envisioned in the original spacecraft design, such as Z-spin thrusting for TDRS-1, can and should be utilized if sufficient risk mitigation can be achieved prior to on-orbit execution. TDRS-1 EOM risk mitigation activities included appropriate analyses, simulations, operational rehearsals, alternate options (where available), and back-out plans to return the spacecraft to a safe, stable condition as required. Key system specifics are listed below.
(1) For key technical issues that require simulation to understand and resolve, utilize independent simulation tools to the extent possible. Significant insight was gained when a MATLAB dynamics simulation was used in addition to the in-house spacecraft simulator.

(2) Rehearse all EOM procedures to the greatest extent possible. Use the rehearsals to validate the EOM procedures as well as train all personnel that will be involved.

(3) Recognize that operational procedures may have conservative constraints imposed that are not appropriate for the EOM phase.

\section{Subsystem}

All subsystems operated in a spacecraft configuration substantially different from the normal mode of operations. EOM subsystem specialists were able to analyze and predict performance in the Z-spin configuration with some degree of confidence using the available subsystem information. The key subsystem lessons learned are listed below:

(1) Characterize the performance of equipment on-orbit that cannot be accurately characterized in ground testing.

(2) Implement a thorough data collection and retention scheme during on-orbit operations that could potentially aid EOM analyses.

(3) Retain spare flight-like equipment (e.g., the TDRS-1 propellant tank) that could be used to predict EOM characteristics.

(4) Dedicate multiple telemetry displays specifically for the EOM flight support team. This avoids conflicts with the needs of the operations team commanding the spacecraft.

(5) Simple but meticulous propellant bookkeeping can result in very accurate knowledge of propellant remaining. TDRS-1 operators achieved an error of $1.2 \%$ over 27 years.

In general subsystem and system designers can take note of the various challenges addressed by the EOM team then incorporate cost-effective features into future designs to increase the level of confidence that EOM requirements for orbital debris compliance will be met, even after on-orbit equipment failures and degradation.

\section{ACRONYMS}

$\begin{array}{ll}\text { ACS } & \text { Attitude Control Subsystem } \\ \text { AGC } & \text { Automatic Gain Control } \\ \text { ATF } & \text { Australian Tracking Data and Relay Satellite } \\ & \text { Facility } \\ \text { CSS } & \text { Coarse Sun Sensor } \\ \text { CPE } & \text { Control Processor Electronics } \\ \Delta v & \text { Delta-Velocity (Orbit Control Maneuver) }\end{array}$




\begin{tabular}{|c|c|}
\hline DOY & Day-Of-Year \\
\hline DTC & Disturbance Torque Compensation \\
\hline DTM & Dual Thruster Module \\
\hline EOM & End-of-Mission \\
\hline EPS & Electrical Power Subsystem \\
\hline FDF & Flight Dynamics Facility \\
\hline FDM & Fill and Drain Module \\
\hline GSFC & Goddard Space Flight Center \\
\hline GEO & Geosynchronous Orbit \\
\hline IMP & Integrated Mission Plan \\
\hline IUS & Inertial Upper Stage \\
\hline JSC & Johnson Space Center \\
\hline MA & Multiple Access \\
\hline MLI & Multi-Layer Insulation \\
\hline NASA & $\begin{array}{l}\text { National Aeronautics and Space } \\
\text { Administration }\end{array}$ \\
\hline NASA HQ & NASA Headquarters \\
\hline NASA STD & NASA Standards \\
\hline NiCad & Nickel Cadmium \\
\hline NPR & NASA Procedural Requirements \\
\hline NSF & National Science Foundation \\
\hline $\mathrm{OTH}$ & Over-the-Horizon \\
\hline PA & Power Amplifier \\
\hline PDM & Propellant Distribution Module \\
\hline RAM & Random Access Memory \\
\hline $\mathrm{RCS}$ & Reaction Control Subsystem (Propulsion) \\
\hline RF & Radio Frequency \\
\hline ROM & Read-Only Memory \\
\hline SA & Single Access \\
\hline SAC & Single Access Compartment \\
\hline SEU & Single Event Upset \\
\hline SGL & Space to Ground Link \\
\hline SSA & S-band Single Access \\
\hline STS & Space Transport System \\
\hline TCS & Thermal Control Subsystem \\
\hline $\mathrm{T} \& \mathrm{C}$ & Telemetry and Command \\
\hline $\mathrm{TT} \& \mathrm{C}$ & Tracking, Telemetry, and Command \\
\hline TDRS & Tracking Data and Relay Satellite \\
\hline TMM & Thermal Math Models \\
\hline TRW & $\begin{array}{l}\text { TRW, Inc was formerly known as } \\
\text { Thompson Ramo Wooldridge Corporation }\end{array}$ \\
\hline TSIM & Tracking Data and Relay Satellite Simulator \\
\hline TWT & Traveling Wave Tube \\
\hline WSC & Whites Sands Complex \\
\hline
\end{tabular}

\section{ACKNOWLEDGMENTS}

The authors wish to acknowledgment the contributions of the following EOM team members to the preparation of this paper: Charles A. Benet, Henry C. Hoffman, Paul G. Kirchman, Thomas E. Williams, and Lawrence A. Woods. They would also like to thank Robert Kilgore and Judith Clark for graphical and editorial support.

\section{REFERENCES}

[1] NPR 8715.6: NASA Procedural Requirements for Limiting Orbital Debris 14 May 2009
[2] Craig Covault, "Loss of TDRS-A Averted by Joint Action," Aviation Week and Space Technology, 11 April 1983

[3] NASA Technical Standard, Process for Limiting Orbital Debris (NASA-STD-8719.14), 6 September 2007

[4] Ralph Jones, "TDRS 1 End of Life Mission Plan Version 2 - ACS/Orbit Perspective," Internal memorandum, September 2009

[5] Ralph Jones, "TDRS 1 End of Mission Propellant Disposal," Internal memorandum, 11 January 2010

[6] C. Chang, N. Dennehy, O. Short, T. Russell, E. Scobey, F. Street, S. Millikin, J. Blue, "TDRS F-1 Launch to Synchronous Orbit Spacecraft Subsystem Performance Report," 31 March 1984

[7] Ralph Jones, "TDRS 1 Orbit Raising During End-ofMission Summary," Memorandum to NASA Code 452, 16 August 2010

[8] White Sands Complex EOM team, "TDRS -1 Integrated End of Mission Plan," 14 June 2010

[9] James Wertz, "Spacecraft Attitude Determination and Control,” page 564, Reidel Publishing Company, 1986.

\section{BIOGRAPHY}

Ron Zaleski is a systems engineer at NASA's Goddard Space Flight Center (GSFC) with over 30 years of experience in electronics design and systems engineering. Mr. Zaleski has held various design and oversight roles while at GSFC including power subsystem engineer overseeing the development of TDRS-8, -9, and-10, as well as design engineer

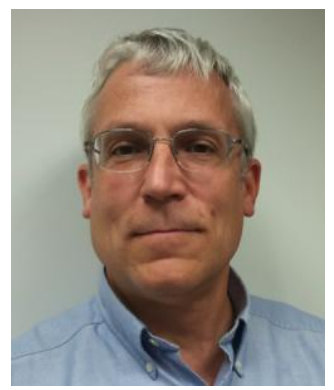
for a number of flight instruments. Prior to his work at GSFC, Mr. Zaleski worked at the Naval Surface Weapons Center, White Oak, as Lead Engineer defining and developing a variety of electronic systems. He is currently responsible for the engineering oversight in managing the long-term viability of the TDRS fleet. He has a Bachelor degree in Electrical Engineering from Drexel University and a Masters degree in Engineering from Johns Hopkins University.

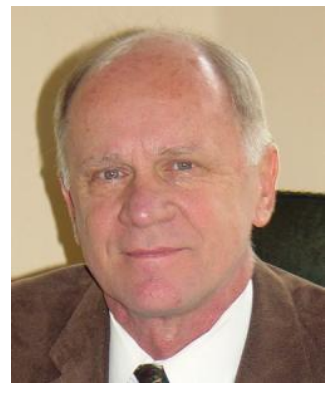

Walt Mirczak is a consultant with 37 years experience in spacecraft operations, systems engineering, and mission analysis. During his career at Northrop Grumman Aerospace Systems, Mr. Mirczak had lead roles on the Prometheus Program, the Earth Observing Satellite Aqua, and the F6 fractionated spacecraft study. Mr. Mirczak was a member of the team that performed ground 
station acceptance testing at White Sands Ground Terminal and supported the launch and rescue efforts of TDRS-1. He remained at White Sands through the launch of TDRS-7. He has a Bachelor degree and Master degree in Aeronautical and Astronautical Engineering from Ohio State University.

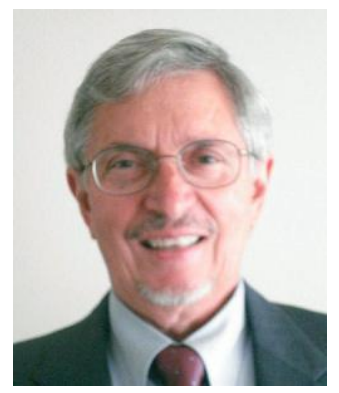

Steve Staich is a consultant with 45 years of experience in spacecraft attitude control, systems engineering, and space vehicle integration. He recently led the integration, test, and launch of the Space Systems Tracking Satellites (STSS) demonstrators. Previously, he led the systems engineering for Earth Observing Satellite Aqua and Aura. In the 1970s he was a member of the original TDRS attitude control design team. In 1983, he was a key contributor to the TDRS rescue effort. He has BSE degrees in Electrical Engineering and Mathematics from University of Michigan, and an MSE in Controls and Computer Design from University of Southern California.

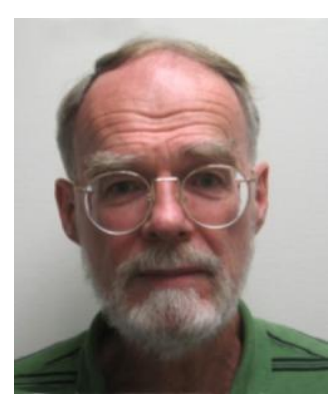

Richard Caverly is a propulsion subsystem engineer at NASA's Goddard Space Flight Center for the TDRS, JWST, and JPSS/NPP programs. Currently, he is with Orbital Science Corporation and has previously worked for Lockheed-Martin Astro-Space (East Windsor, NJ) and the Boeing Satellite Systems (El Segundo, CA). He has a Master of Science degree in Meteorology from the Pennsylvania State University.

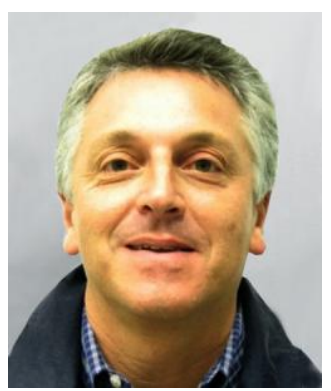

Nick Teti is a senior thermal engineer with 25 years experience in spacecraft thermal systems engineering. Mr. Teti has held lead thermal engineering roles on the Earth Observing-1 spacecraft and Spartan 207/IAE mission for NASA/GSFC and the thermal engineering manager for ATK Space (formerly Swales Aerospace). In 2002, Mr. Teti joined the TDRS sustaining engineering team to provide thermal engineering oversight for the current TDRS on-orbit fleet of satellites. In addition, Mr. Teti is currently the Observatory Thermal Manager for the NASA/GSFC Magnetospheric MultiScale (MMS) mission scheduled for launch in 2014 and is supporting the TDRS K/L continuation effort as a member of the independent review team. He has a Bachelor degree in Mechanical Engineering from the University of Maryland.

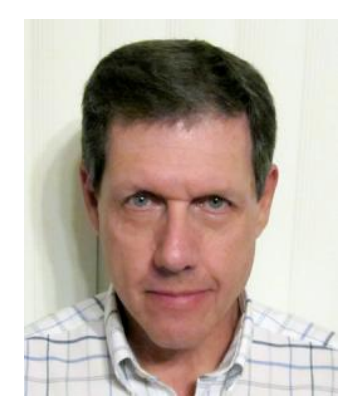

W. L. Vaught is a senior spacecraft systems engineer with 30 years of experience in space systems engineering and space operations. Mr. Vaught served as the lead TDRS 1 EOM engineer. He has been a flight control team systems engineer for legacy and second generation TDRS spacecraft launches, served in a variety of Test Director roles, and has led many system design development projects. Prior to the TDRS project he served the USAF DMSP project and other USAF missions and as a Space Systems Director. He is presently assigned to the NASA TDRS Project Spacecraft Engineering Group with General Dynamics C4 Systems where he continues to serve in lead roles. He has a Bachelor of Science degree in Physics from Missouri State University and a Master of Business Administration degree from Webster University.

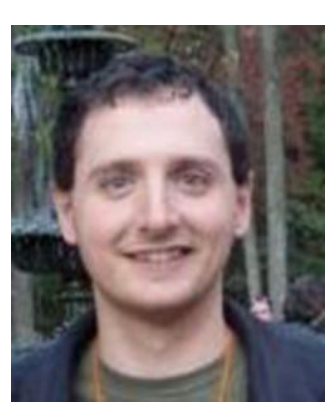

Eric Smith led the Flight Dynamics Facility in support of TDRS-1 EOM activities. He has a Bachelor of Science degree in Aerospace Engineering from the University of Maryland and has been working in the orbit determination and tracking data evaluation groups at GSFC's Flight Dynamics Facility since June 2007.

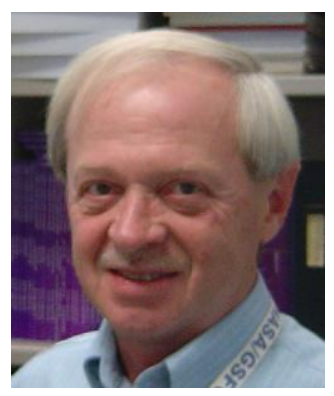

Dave Olney has been attitude control systems engineer at the GSFC for 41 years. During that time he has provided attitude control analysis for a variety of science missions on sounding rockets, stratospheric balloons, space shuttle, and scientific satellites. He has also supported multiple design studies at the Mission Design Laboratory at GSFC. He holds a Bachelor degree in Aerospace Engineering from the University of Cincinnati and a Masters degree in Engineering Management from the George Washington University. 